Z. klin. Chem. u. klin. Biochem.

8. Jg., S. $637-648$, November 1970

\title{
Bestimmung der freien Katecholamine im Harn
}

\author{
Von H. WISSER \\ Max-Planck-Institut für Psycbiatrie, München
}

(Eingegangen am 31. Juli 1970)

\begin{abstract}
Es wird über ein teilautomatisches Bestimmungsverfahren von Adrenalin und Noradrenalin im Urin berichtet. Dazu wurde das Verfahren von Weil-Malmerbe und Bigelow $(1,2)$ mit geringen Änderungen auf den AutoAnalyzer adaptiert. Der Einfluß der Kupferionen bei der Bestimmungsreaktion wurde untersucht. Die Zuverlässigkeitskriterien der Methode wurden ermittelt. Verschiedene Parameter des Abtrennverfahrens von CROuT (3) wurden geprüft. Es wurden Untersuchungen zur Verwahrung von Säuleneluaten durchgeführt. Die 24-Stdn-Ausscheidung von Adrenalin, Noradrenalin, Gesamtkatecholaminen und Dopamin wurde bei einer Stichprobe von 47 Frauen und 51 Männern unter statistischer Qualitätskontrolle bestimmt. Die Produkt-Moment-Korrclationskoeffizienten für die einzelnen GröBen untereinander wurden berechnet. Es wird über die Ausscheidung der genannten Verbindungen sowie der Vanillinmandelsäure im 24-Stdn-Urin eines Patienten mit einem operativ gesicherten Phäochromocytom berichtet.
\end{abstract}

\section{The determination of free catecholamines in urine}

A semiautomatic method is reported for the determination of adrenaline and noradrenaline in urine. The method of WEIL-MALHERBE and Bigelow $(1,2)$ has been slightly modified and adapted for the AutoAnalyzer. The effect of copper ions on the determination method was investigated. The criteria of reliability for the method are reported. Different parameters of the separation method of CRour (3) were tested. Studies were made on the preservation of column eluates. The 24 . hr excretion of adrenaline, noradrenaline, total catecholamines and dopamine was determined with statistical quality control on a random sample of 47 women and 51 men. The correlation coefficients were calculated for the individual values as a group. The excretion of the above compounds and vanillinmandelic acid is reported in the $24 \mathrm{hr}$ urine of a patient with an operatively confirmed phaeochromocytoma.

Unter dem Sammelbegriff Katecholamine werden die Verbindungen Adrenalin, Noradrenalin und Dopamin ${ }^{1}$ ) zusammengefaßt. Diese Substanzen werden in unterschiedlicher Menge im 24-Stunden-Urin ausgeschieden. Die quantitative Bestimmung im Urin wird meist nach Abtrennung an Aluminiumoxid oder Ionenaustauschern - fluorometrisch mit der Trihydroxiindolmethode durchgeführt. Die erwähnte Abtrennung ist eine Reinigungschromatographie und führt nicht zu einer Auftrennung in die einzelnen Verbindungen. Eine vollständige Auftrennụng in die Einzelkomponenten, z. B. mit Ionenaustauschern, ist so zeitaufwendig (4), $\mathrm{da} ß$ man bei der differentiellen Bestimmung darauf verzichtet. Die spektralen Eigenschaften des Dopaminfluorophors gestatten eine Bestimmung von Dopamin neben Adrenalin und Noradrenalin. Zur differentiellen Adrenalin- und Noradrenalinbestimmung werden Unterschiede der optischen Eigenschaften der Fluorophore $(5,6,7)$, des stabilisierenden Einflusses verschiedener Reduktionsmittel $(8,9,10,11)$, sowie die $\mathrm{pH}$-Abhängigkeit der Oxydation benutzt $(3,7,12)$.

Das erste teilautomatische Bestimmungsverfahren für Adrenalin und Noradrenalin wurde von MERrILLs eingeführt $(8,9)$. Diese von verschiedenen Autoren $(13,14$, 15) angewandte Methode hat den Nachteil, daß die kleinste Größe, das Adrenalin, aus der Differenz zweier großer Meßwerte, den Gesamtkatecholaminen und dem Noradrenalin, ermittelt wird. Das Gleiche gilt auch für ein ähnliches Verfahren von VrkToRIA und Mitarbeitern (16). Von MABRY und WARTH (17) wurde eine teilautomatische Bestimmung mit Oxydation bei zwei ver-

1) Dopamin $=0$-Dihydroxyphenyläthylamin, Dopa $=0$-Dihydroxyphenylalanin. schiedenen $\mathrm{pH}$-Werten durchgeführt, wobei Adrenalin durch Oxydation bei $\mathrm{pH}$ 3,5 und Ascorbatzugabe bestimmt wird. Nachteilig bei dieser Methode ist, daß die Fluoreszenzintensität des Adrenalins nach Oxydation bei saurem pH-Wert mit Kaliumhexacyanoferrat-[III] und anschließendem Zusatz einer alkalischen Lösung von Ascorbinsäure oder Dimercaptopropanol niedrig ist (2). Führt man die Oxydation bei $\mathrm{pH} 2,8$ unter Zusatz von Kupferionen durch, wobei 2-Mercaptoäthanol als Reduktionsmittel benutzt wird, so erhält man nach WEILMalmerbe und Bigelow (1) eine erheblich größere Fluoreszenzausbeute. Diese Erhöhung der Fluoreszenzausbeute durch Kupferionen wurde auch von anderer Seite bestätigt (18). Diese für die Adrenalinbestimmung günstigen Bedingungen - differentielle Bestimmung durch Oxydation bei zwei verschiedenen $\mathrm{pH}$-Werten und hohe Fluoreszenzausbeute - waren der Grund, das Verfahren von WeIL-MalHerbe und Bigelow für eine Automation auszuwählen. Die Automation der Bestimmung hat den Vorteil, daß die Gefahr der Einschleppung von Störsubstanzen durch Glasgeräte vermindert und die zeitliche Konstanz der Abfolge von Reagenzienzugabe und Fluoreszenzmessung („timing“) gewährleistet ist. Außerdem ist das Verfahren weniger arbeitsintensiv. Dieser letzte Punkt macht sich besonders bei größeren Meßreihen bemerkbar.

\section{Methodik}

Die Methode der Dopaminbestimnung wurde schon in dieser Zeitschrift veröffentlicht $\left.(19)^{2}\right)$, so daß auf die dort angegebenen Daten

$\left.{ }^{2}\right)$ In dem dort angegebenen Flie(3schema ist ein Fehler. Uber die Position 2 wird die Probc $\left(0,035^{\prime \prime}\right)$ und Position $4\left(0,045^{\prime \prime}\right)$ der Puffer zugeführt. 
verwiesen wird. Für die Bestimmung der übrigen Meßgrößen wird nochmals eine ausführliche Beschreibung gegeben ${ }^{3}$ ). Gegenüber dem Verfahren von WeIL-MALiHerbe $(1,2)$ sind folgende geringfügige Änderungen vorgenommen worden. Als Reduktionsmittel wird nur eine wäßr. 2-Mercaptoäthanol-Lösung ohne Natriumsulfit-Zusatz benutzt. Außerdem wird die Probe nach Zusatz der Thiolat-Lösung nicht auf pH 5,0 eingestellt. Das Ansäuern der Probe führt zu ciner Zunahme der Stabilität des Fluorophors. Bei einer semiautomatischen Bestimmungsmethode mit einem exakten „timing“ ist dies nicht unbedingt notwendig. Außerdem fällt in Anwesenheit von Kupfer nach dem Ansäuern ein weißer Niederschlag (1) aus, der bei einem kontinuierlichen Bestimmungsverfahren durch Einbeziehung eines Dialyseschrittes entfernt werden müßte. Es sei vorweggenommen, daß für die Adrenalin-, Noradrenalin- und Gesamtkatecholaminbestimmung das gleiche Fließschema (Abb. 1) benutzt wird. Bei der Analyse des Normalkollektivs waren die Volumina so gewählt, $\mathrm{da} ß$ nach Aliquotierung der Probe und Einstellen des pH-Wertes auf 2,8 bzw. 6,0 aus dem letzteren Teil für jede Urinprobe Noradrenalin, Gesamtkatecholamine und Dopamin bestimmt werden konnten.

Adrenalin wird bei pH 2,8 mit Kaliumhexacyanoferrat-[III] unter Zusatz von Kupferionen oxydiert, Noradrenalin bei pH 6,0 ohne Kupferionenzusatz. Die Bildung der Fluorophore erfolgt in alkalischer 2-Mercaptoäthanol-Lösung.

Zur Bestimmung der Gesamtkatecholamine vird das auf pH 6,0 eingestellte Eluat mit Kaliumhexacyanoferrat-[III] oxydiert. Die Fluorophorbildung wird durch Zusatz einer alkalischen Ascorbinsäurelösung ermöglicht.

Dopamin wird durch Oxydation mit Natrium-meta-perjodat und anschließendes Zufügen einer alkalischen Natriumsulfitlösung in die fluoreszierende Verbindung überführt.

\section{Fluorometrische Messungen}

\section{Apparate und Glasgefäße}

1. Der AutoAnalyzer ${ }^{4}$ ) wird in Verbindung mit einem Turner Fluorometer Modell 111 mit Durchflußküvettentür ${ }^{5}$ ) benutzt. Für den Anschluß des Bristol-Zweikanalschreibers ist ein besonderer Adapter ${ }^{5}$ ) erforderlich. Als Lichtquelle des Fluorometers wird eine Quecksilberlampe $\left.(110-850)^{5}\right)$ benutzt. Das Primärfilter ist ein Bandfilter $\left.(110-813)^{5}\right)$ mit einem Durchlaßmaximum bei $436 \mathrm{~nm}$ und das Sekundärfilter $\left.(110-826)^{5}\right)$ ein Kantenfilter mit einer Kante bei $520 \mathrm{~nm}$.

2. Autotitrator Typ TTT 1c in Verbindung mit der Autobürette $A B U 1^{6}$ ) und den Elektroden G $202 \mathrm{C}$ und $\mathrm{K}$ 401, sowie GK $\left.2321 \mathrm{C}^{6}\right)$.

3. Chromatographierohre $30 \mathrm{~cm}$ lang, $1 \mathrm{~cm}$ Durchmesser, vor dem Ablauf eine eingeschmolzene G-2-Fritte, Teflonküken.

\section{Reagenzien}

Wenn nicht anders vermerkt, werden p. a. Reagenzien der Firma Merck, Darmstadt, benutzt.

\section{Abtrennung}

1. Aluminiumoxid Woelm neutral ${ }^{7}$ ) (nach Vorschrift von CROUT (3) behandelt).

2. $0,2 \mathrm{M}$ Äthylendiamintetraessigsäure (EDTA).

3. $\mathrm{NaHCO}_{3} / \mathrm{Na}_{2} \mathrm{CO}_{3}$-Lösung (je $80 \mathrm{~g} / l$ ).

4. 0,2Mr Essigsäure.

3) Teile dieser Arbeit wurden auf der Tagung Biochemische Analytik vorgetragen (20).

4) Hersteller: Technicon Instruments Corpor. Chauncey, USA. Deutsche Vertretung: Technicon GmbH, Frankfurt/Main.

$\left.{ }^{5}\right)$ Hersteller: G. K. Turner Assoc., Palo Alto, USA. Vertretung: Camag, Muttenz, Schweiz.

6) Hersteller: Radiometer, Kopenhagen, Dänematk: Deutsche Vertretung: $\mathrm{K}$. Hillerkus, Krefeld.

7) M. Woelm, Eschwege.

\section{Adrenalinbestimmung}

5. 2N Ameisensäure.

6. Natriumformiatpuffer $\mathrm{pH} 2,8(0,2 \mathrm{~N} \mathrm{NaOH}$ und $0,2 \mathrm{~N}$ Ameisensäure $1 / 7(\mathrm{v} / \mathrm{v}))$.

7. 0,5 proz. Kaliumhexacyanoferrat-[III]-Lösung ( $5 \mathrm{~g}$ in 1 Liter Lösung 6).

8. 0,2 proz. Kupferacetatmonohydrat-Lösung $(2 \mathrm{~g}$ in 1 Liter Lösung 6).

9. 2,0proz. wäßr. 2-Mercaptoäthanol-Lösung $\left.{ }^{8}\right)(v / v)$.

10. $10 \mathrm{~N} \mathrm{NaOH}$.

\section{Noradrenalinbestimmung}

11. $2 \mathrm{~N} \mathrm{KOH}$.

12. $0,2 \mathrm{M}$ Kaliumacetatpuffer $\mathrm{pH} 6,0(19,6 \mathrm{~g}$ Kaliumacetat $/ l$, mit Eisessig auf $\mathrm{pH}$ 6,0 einstellen).

13. 0,5proz. Kaliumhexacyanoferrat-[III]-Lösung (5 $\mathrm{g}$ in 1 Liter Lösung 12).

14. $5 \mathrm{~N} \mathrm{NaOH}$

(Reduktionsmittel s. Adrenalinbestimmung).

Gesamtkatecholaminbestimmung

gleiche Reagenzien wie bei der Noradrenalinbestimmung.

15. 0,5proz. Ascorbinsäurelösung (als Reduktionsmittel).

Standards

16. Stammlösung Adrenalin: $18,2 \mathrm{mg}$ Adrenalin-hydrogentartrat $\left.^{8}\right)$ in $10 \mathrm{~m} l 0,1 \mathrm{~N} \mathrm{HCl}$

17. Stammlösung Noradrenalin: $19,9 \mathrm{mg}$ Noradrenalin-hydrogentartratmonohydrat ${ }^{9}$ ) in $10 \mathrm{ml} 0,1 \mathrm{~N} \mathrm{HCl}$.

18. Arbeitslösungen: Verdünnungen der Stammlösungen mit demin. Wasser 1:100.

Arbeitsweise

Abtrennung

Zur Analyse werden 5 Prozent des 24-Stdn-Urins eingesetzt. Die Probe wird zentrifugiert und die entsprechende Menge in ein $200 \mathrm{ml}$ Titriergefäß der Aurobürette einpipettiert. Anschließend werden $5 \mathrm{ml} 0,2 \mathrm{M}$ EDTA-Lösung und $3 \mathrm{~g} \mathrm{Al}_{2} \mathrm{O}_{3}$ mit einem Meßlöffel zugegeben. Mit Lösung 3 wird die Probe am Autotitrator auf $\mathrm{pH}$ 8,5 eingestellt. Nach Einstellung des $\mathrm{pH}$-Wertes wird die Probe zur Vervollständigung der Adsorption noch 10 Min. mechanisch getührt. Es wurde eine Vorrichtung gebaut, mit der drei Proben gleichzeitig gerührt werden können. Nach dem Rühren läßt man die Probe 3-5 Min. stehen, bis das Aluminiumoxid sich abgesetzt hat. Der Uberstand wird dann mit der Wasserstrahlpumpe vorsichtig abgezogen. Der Rückstand wird mit etwas demin. Wasser aufgenommen und möglichst quantitativ in eine Chromatographiesäule überführt. Das Aluminiumoxid wird mit $30 \mathrm{~m} l$ demin. Wasser nachgewaschen. Dann werden $3 \mathrm{ml} 0,2 \mathrm{M}$ Essigsäure auf die Säule gegeben. Dieses Eluat wird verworfen. Die Elution der Katecholamine wird mit $8 \mathrm{ml} 0,2 \mathrm{M}$ Essigsäure durchgeführt. Das Eluat wird in einem auf $10 \mathrm{~m} /$ Einguß geeichten Zentrifugenglas gesammelt. Die Eluate werden mit demin. Wasser auf $10 \mathrm{~m} /$ aufgefüllt und zentrifugiert. In jeweils neue Gefäße werden $4 \mathrm{~m} l$ für die Adrenalinbestimmung und $4 \mathrm{~m} l$ für die Noradrenalinbestimmung pipettiert. Anschließend werden sie am Autotitrator mit 2N KOH auf $\mathrm{pH} 6,0$ bzw. mit 2N Ameisensäure auf $\mathrm{pH} 2,8$ eingestellt. Die Proben für die Adrenalinbestimmung werden mit Natriumformiatpuffer $\mathrm{pH} 2,8$ und die Proben für die Noradrenalinbestimmung mit Kaliumacetatpuffer $\mathrm{pH} 6,0$ auf $5 \mathrm{ml}$ aufgefüllt. Bei der Bestimmung für die Normalwerte wurde aus dem gleichen Eluat noch die Gesamtkatecholamine und das Dopamin bestimmt.

\section{Bestimmung}

Das Fließschema des AutoAnalyzers für die fluorometrische Bestimmung des Adrenalins ist in Abbildung 1 wiedergegeben. Dic

8) Serva Entwicklungslabor, Heidelberg.

9) Fluka AG, Buchs (Schweiz). 


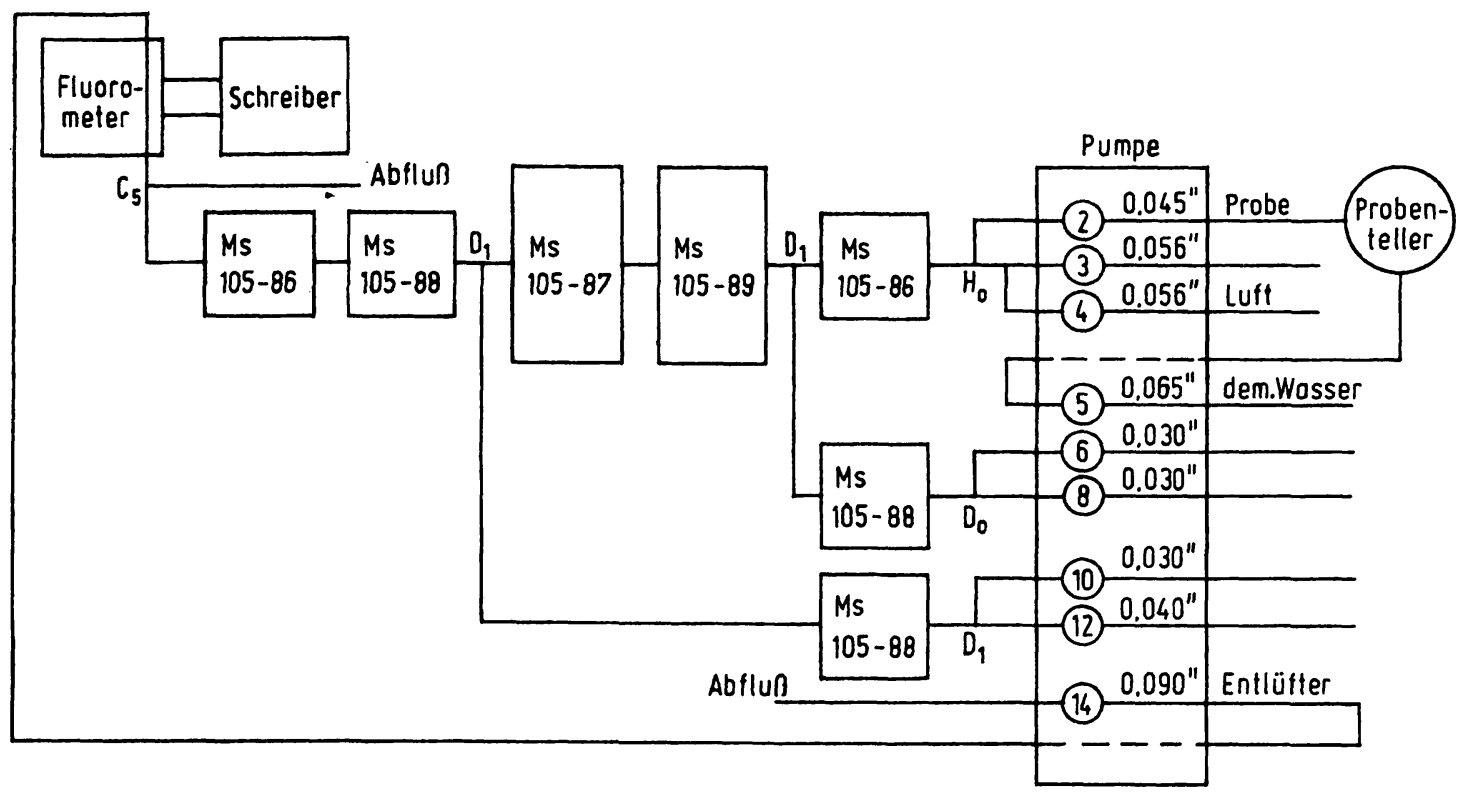

\begin{tabular}{|c|c|c|c|c|c|}
\hline Bestandteil & 3 & 6 & Lösungen in den Positionen & 10 & 12 \\
\hline Adrenalin & $\begin{array}{l}\text { Puffer } \\
\text { (6) }\end{array}$ & $\begin{array}{l}\text { 0,2proz. } \\
\text { Kupferacetatlösung } \\
\text { (8) }\end{array}$ & $\begin{array}{l}\text { 0,5proz. } \\
\text { Kaliumhexacyano- } \\
\text { ferrat-(III)-Lösung } \\
\text { (7) }\end{array}$ & $\begin{array}{c}\text { 2proz. } \\
\text { 2-Mercapto- } \\
\text { äthanollosung } \\
(9)\end{array}$ & $\begin{array}{c}10 \mathrm{~N} \mathrm{NaOH} \\
(10)\end{array}$ \\
\hline Noradrenalin & $\begin{array}{l}\text { Puffer } \\
\text { (12) }\end{array}$ & $\begin{array}{l}\text { Puffer } \\
\text { (12) }\end{array}$ & $\begin{array}{l}\text { 0,5proz. } \\
\text { Kaliumhexacyano- } \\
\text { ferrat-(1II)-Lösung } \\
\text { (7) }\end{array}$ & $\begin{array}{l}\text { 2proz. } \\
\text { 2-Mercapto- } \\
\text { äthanollosung } \\
\text { (9) }\end{array}$ & $\begin{array}{c}5 \mathrm{~N} \mathrm{NaOH} \\
(14)\end{array}$ \\
\hline Gesamtkatecholamine & $\begin{array}{l}\text { Puffer } \\
\text { (12) }\end{array}$ & $\begin{array}{l}\text { Puffer } \\
\text { (12) }\end{array}$ & $\begin{array}{l}\text { 0,5proz. } \\
\text { Kaliumhexacyano- } \\
\text { ferrat-(III)-Lósung } \\
\text { (7) }\end{array}$ & $\begin{array}{c}\text { 0,5proz. } \\
\text { Ascorbinsäurelösung } \\
\text { (15) }\end{array}$ & $\begin{array}{c}5 \mathrm{~N} \mathrm{NaOH} \\
\text { (14) }\end{array}$ \\
\hline
\end{tabular}

Abb. 1

Fließschema des AutoAnalyzers für die Adrenalin-, Noradrenalin- und Gesamtkatecholaminbestimmung. Die Ziffernangaben sind die Innendurchmesser der Pumpenschläuche in inch. Die eingekreisten Zahlen geben ihre Position auf der Pumpe an. $H_{0}$, $D_{0}, D_{1}$ und $C_{5}$ sind die Bezeichnungen des Herstellers für die Fittings. MS bedeutet Mischspirale. Die dazugehörigen Ziffern sind die entsprechenden Katalognummern. Die Lösungen, die über die Positionen $3,6,8,10$ und 12 zugeführt werden, sind für die jeweilige Bestimmung tabellarisch zusammengefaßt. Die in Klammern gesetzten Zahlen bezeichnen die Position der jeweiligen Lösung mit Reagenzienverzeichnis

Anordnung der Mischschlangen ist bei der Bestimmung von Adrenalin und Noradrenalin gleich. Alle Leitungen sind in Glas verlegt. Bei der Noradrenalinbestimmung ergeben sich gegenüber der Adrenalinbestimmung folgende Änderungen. In Position 3 und 8 (A'bb. 1) wird anstatt des Natriumformiatpuffers bzw. der Kupferacetatlösung 0,2 M Kaliumacetatpuffer $\mathrm{pH}$ 6,0 zugeführt. Außerdem wird die $10 \mathrm{~N} \mathrm{NaOH}$ durch $5 \mathrm{~N} \mathrm{NaOH}$ ersetzt. Bei der Gesanıtkatecholaminbestimmung werden mit einer einzigen Ausnahme die gleichen Reagenzien benutzt, wie bei der Noradrenalinbestimmung. Anstelle der 2proz. 2-Mercaptoäthanollösung wird 0,5proz. Ascorbinsäurelösung als. Reduktionsmittel zugesetzt. Die Analysengeschwindigkeit beträgt 40 oder 60 Proben pro Stunde. Allerdings befindet sich zwischen zwei Proben ein Gefäß mit demin. Wasser. Die resultierende Fluoreszenzz wird über einen Schreiber registriert. Die quantitative Auswerțung erfolgt über die Peakhöhen.

Erwähnt sei, daß auch ein Fließschema ohne Verdünnung der Probe mit Puffer benutzt wurde. Gegenüber dem in Abbildung 1 gezeigten Fließschema ergeben sich dann folgende Änderungen. Der Probenschlauch $\left(0,045^{\prime \prime}\right)$ wird durch einen Schlauch mit einem Innendurchmesser von 0,073" ersetzt. Allerdings ist dann der Einbau eines Entlüftungsfittings exforderlich. Es entfällt die Spirale zur Mischung von Probe und Puffer.

Die Konzentrationsbestimmung der Proben erfolgt über drci externe Standards. Bei jeder Versuchsreihe werden zwischen 30 und $200 \mu l$ Arbeitslösung (je nach der erforderlichen Spaltbrcite des Fluorometers) zu $9 \mathrm{~m} l 0,2 \mathrm{M}$ Essigsäure pipettiert und mit demin. Wasser auf $10 \mathrm{~m} /$ aufgefüllt. Diese Standardlösungen werden dann wie die Proben nach der Elution weiterbehandelt. Bei der Adrenalinbestimmung wird ein Noradrenalinstandard mitgeführt, um den
Noradrenalineinfluß bei der Adrenalinbestimmung berücksichtigen zu können.

Die Leerwerte werden so crmittelt, daß nach Ersatz der 2-Mercaptoäthanollösung durch demin. Wasser die Fluoreszenz erneut gemessen wird.

Berechnung:

$$
\begin{aligned}
& \mu \mathrm{g} \mathrm{NA} / \text { Eluat }=\frac{\operatorname{cST}_{\mathrm{ST}}\left(\mathrm{P}_{\mathrm{P}}-\mathrm{P}_{\mathrm{L}}\right)}{\mathrm{P}_{\mathrm{ST} / \mathrm{NA}}} \\
& \mu \mathrm{g} \quad \mathrm{A} / \text { Eluat }=\frac{\left(\mathrm{P}_{\mathrm{P}}-\mathrm{P}_{\mathrm{L}}\right)-\mu \mathrm{g} \mathrm{NA} / \text { Eluat } \times \mathrm{K}_{1}}{\mathrm{~K}_{2}}
\end{aligned}
$$

$P_{P}=$ Peakhöhe der Probe

$P_{L}=$ Peakhöhe des Leenwertes

$P_{\mathrm{ST}}=$ Peakhöhe des Standards

$c_{\mathrm{ST}}=$ Konzentration des Standards $\mu \mathrm{g} / 10 \mathrm{inl}$

$$
\begin{aligned}
& K_{1}=\frac{P_{S T / N A}}{c_{S T / N A}} \\
& K_{2}=\frac{P_{S T / A}}{c_{S T / A}}
\end{aligned}
$$

$\mathrm{NA}=$ Noradrenalin

$A=$ Adrenalin

\section{Radiocbenische Messungen}

Ein Teil der Versuche zur Uberprüfung des Abtrennverfahrens wurde radiochemisch durchgeführt. Zur Bestimmung der Aktivität von ${ }^{14} \mathrm{C}$-markicrtem Adrenalin, Noradrenalin und Dopamin ${ }^{10}$ )

10) Hersteller: NEN Corp. Boston, USA. Deutsche Vertretung: NEN Chemicals Gnbbll, Drcieichenhain, Frankfurt/Main. 
wurde das Liquid-Szintillation-Spektrometer Modell 3375'11) benutzt. Die Reinheit der genannten Verbindungen wurde dünnschichtchromatographisch geprüft. In der benutzten Meßanordnung ergaben $0,02 \mu \mathrm{g}$ Adrenalin, $0,02 \mu \mathrm{g}$ Noradrenalin und $0,4 \mu \mathrm{g}$ Dopamin etwa $2 \cdot 10^{4} \mathrm{Imp}$./Min. Der Nulleffekt lag bei 20-30 Imp./Min. Zur Messung wurden $1-3 \mathrm{~m} l$ der essigsauren Eluate nach $\mathrm{Al}_{2} \mathrm{O}_{3}$-Chromatographie mit Szintillationsflüssigkeit nach $\mathrm{HAYES}^{12}$ ) auf $20 \mathrm{~m} /$ aufgefüllt und die Aktivität bestimmt. Die Auswertung erfolgte nach der Methode des inneren Standards.

\section{Zuverlässigkeitskriterien der Methode}

Dieser methodische Teil ist kurz gefaßt, da die Daten teilweise schon in vorhergehenden Arbeiten publiziert wurden $(19,20)$.

\section{Präzision}

Die Analysen der Proben des Normalkollektivs wurden unter statistischer Qualitätskontrolle durchgeführt. Dazu wurden bei jeder Serie Doppelbestimmungen mit selbst hergestellten Kontrollurinproben durchgeführt. Aus der Differenz der Doppelbestimmungen wurde die Streuung in der Serie und aus dem ersten Wert der

11) Hersteller: Packard Instrument Corp., Inc. Downers Grove, USA. Deutsche Vertretung: Packard Instruments GmbH, Frankfurt/Main.

12) Packard Technical Bulletin, Nr. 1 April (1963).
Doppelbestimmungen die Streuung von Tag zu Tag berechnet. Außerdem wurde zu einem späteren Zeitpunkt aus Neunfachbestimmungen derselben Kontrollprobe die Streuung in einer Serie berechnet. Die Ergebnisse sind in Tabelle 1 zusammengefaßt.

Der Variationskoeffizient für die Streuung in der Serie aus $\mathrm{n}$-fach-Bestimmungen ist bei allen Bestandteilen am kleinsten, während er für die Streuung von Tag zu Tag am größten ist. Die bei der Ermittlung der Streuung in der Serie aus den Neunfachbestimmungen gewonnenen arithmetischen Mittelwerte liegen fast alle niedriger als die aus den Doppelbestimmungen ermittelten Werte, aber noch innerhalb der 3s-Grenze.

\section{Wiederfindung}

Die Wiederfindungsrate wurde durch Aufstockungsversuche unterschiedlicher Größe an Poolurinproben bestimmt. Die für die Fluorometrie angegebenen Werte sind die Spannweiten der ermittelten Wiederfindungen für unterschiedliche Aufstockzusätze aus verschiedenen Versuchsreihen im Laufe eines Jahres (letzte Spalte Tabelle 2). Die radiochemisch ermittelten Ausbeuten sind Mittelwerte aus Dreifachbestimmungen (vorletzte Spalte Tabelle 2).

Tab. 1

Ergebnis der Präzisionskontrolle mit selbst hergestellten Kontrollurinproben

\begin{tabular}{|c|c|c|c|c|}
\hline Bestandteil & $\begin{array}{l}\text { statistische } \\
\text { Kenngröße }\end{array}$ & $\begin{array}{c}\text { in der Serie aus } \\
n \text {-fach-Bestimmungen }\end{array}$ & $\begin{array}{c}\text { in der Serie aus } \\
\text { Doppelbestimmungen }\end{array}$ & von Tag zu Tag \\
\hline Adrenalin & $\begin{array}{l}\overline{\mathrm{x}}(\mu \mathrm{g} / l) \\
\mathrm{s}(\mu \mathrm{g} / l) \\
\mathrm{V}(\%) \\
\mathrm{n}\end{array}$ & $\begin{array}{c}7,8 \\
0,3 \\
4,5 \\
\text { Neunfachbestimmungen }\end{array}$ & $\begin{array}{c}8,9 \\
0,6 \\
6,3 \\
18 \text { Doppelbestimmungen }\end{array}$ & $\begin{array}{c}8,8 \\
0,7 \\
7,8 \\
20 \text { Einfachbestimmungen }\end{array}$ \\
\hline Noradrenalin & $\begin{array}{l}\overline{\mathrm{x}}(\mu \mathrm{g} / l) \\
\mathrm{s}(\mu \mathrm{g} / l) \\
\mathrm{V}(\%) \\
\mathrm{n}\end{array}$ & $\begin{array}{c}38 \\
1,5 \\
3,9 \\
\text { Neunfachbestimmungen }\end{array}$ & $\begin{array}{c}49 \\
3 \\
6,7 \\
18 \text { Doppelbestimmungen }\end{array}$ & $\begin{array}{c}50 \\
6 \\
12,3 \\
20 \text { Einfachbestimmungen }\end{array}$ \\
\hline Gesamtkatecholamine & $\begin{array}{l}\overline{\mathrm{x}}(\mu \mathrm{g} / l) \\
\mathrm{s}(\mu \mathrm{g} / l) \\
\mathrm{V}(\%) \\
\mathrm{n}\end{array}$ & $\begin{array}{c}67 \\
1,4 \\
2,1 \\
\text { Neunfachbestimmungen }\end{array}$ & $\begin{array}{c}73 \\
2 \\
2,2 \\
19 \text { Doppelbestimmungen }\end{array}$ & $\begin{array}{c}73 \\
5 \\
7,1 \\
21 \text { Einfachbestimmungen }\end{array}$ \\
\hline Dopamin & $\begin{array}{l}\overline{\mathrm{x}}(\mu \mathrm{g} / l) \\
\mathrm{s}(\mu \mathrm{g} / l) \\
\mathrm{V}(\%) \\
\mathrm{n}\end{array}$ & $\begin{array}{c}290 \\
8 \\
2,9 \\
\text { Neunfachbestimmungen }\end{array}$ & $\begin{array}{c}281 \\
13 \\
4,5 \\
19 \text { Doppelbestimmungen }\end{array}$ & $\begin{array}{c}281 \\
19 \\
6,9 \\
21 \text { Einfachbestimmungen }\end{array}$ \\
\hline
\end{tabular}

Tab. 2

Ergebnis von Wiederfindungsversuchen an Poolurinproben

\begin{tabular}{|c|c|c|c|c|c|c|c|}
\hline Bestandteil & $\begin{array}{l}\text { statistische } \\
\text { Kenngröße }\end{array}$ & $\begin{array}{l}\text { nicht auf- } \\
\text { gestockt }\end{array}$ & $\begin{array}{l}\text { auf- } \\
\text { gestockt }\end{array}$ & $\begin{array}{l}\text { Aufstock- } \\
\text { zusatz ( } \mu \mathrm{g})\end{array}$ & $\begin{array}{c}\text { Ausbeute } \\
\%\end{array}$ & $\begin{array}{c}\text { Ausbet } \\
\text { radiochemisch }\end{array}$ & $\begin{array}{l}\text { ute } \% \\
\text { fluorometrisch }\end{array}$ \\
\hline Adrenalin & $\begin{array}{c}\overline{\mathrm{x}}(\mu \mathrm{g} / 40 \mathrm{ml}) \\
\mathrm{s}(\mu \mathrm{g} / 40 \mathrm{ml}) \\
\mathrm{V}(\%) \\
\mathrm{n}\end{array}$ & $\begin{array}{l}0,30 \\
0,005 \\
1,8 \\
5\end{array}$ & $\begin{array}{l}0,54 \\
0,016 \\
2,9 \\
5\end{array}$ & 0,3 Adrenalin & $\begin{array}{r}80 \\
5 \\
5\end{array}$ & $\begin{array}{l}83 \\
3\end{array}$ & $78-90$ \\
\hline Noradrenalin & $\begin{array}{c}\overline{\mathrm{x}}(\mu \mathrm{g} / 40 \mathrm{ml}) \\
\mathrm{s}(\mu \mathrm{g} / 40 \mathrm{ml}) \\
\mathrm{V}(\%) \\
\mathrm{n}\end{array}$ & $\begin{array}{l}2,14 \\
0,08 \\
3,8 \\
5\end{array}$ & $\begin{array}{l}3,82 \\
0,06 \\
1,6 \\
5\end{array}$ & 2,0 Noradrenalin & $\begin{array}{r}84 \\
3 \\
5\end{array}$ & $\begin{array}{l}84 \\
3\end{array}$ & $79-88$ \\
\hline Gesamtkatecholamine & $\begin{array}{c}\overline{\mathrm{x}}(\mu \mathrm{g} / 40 \mathrm{ml}) \\
\mathrm{s}(\mu \mathrm{g} / 40 \mathrm{ml}) \\
\mathrm{V}(\%) \\
\mathrm{n}\end{array}$ & $\begin{array}{l}2,89 \\
0,11 \\
3,8 \\
5\end{array}$ & $\begin{array}{l}4,32 \\
0,02 \\
0,5 \\
5\end{array}$ & 2,0 Noradrenalin & $\begin{array}{r}71 \\
1 \\
5\end{array}$ & $\begin{array}{l}84 \\
3\end{array}$ & $72-80$ \\
\hline Dopamin & $\begin{array}{c}\overline{\mathrm{x}}(\mu \mathrm{g} / 40 \mathrm{ml}) \\
\mathrm{s}(\mu \mathrm{g} / 40 \mathrm{ml}) \\
\mathrm{V}(\%) \\
\mathrm{n}\end{array}$ & $\begin{array}{l}7,6 \\
0,44 \\
5,9 \\
5\end{array}$ & $\begin{array}{c}13,0 \\
0,44 \\
3,4 \\
5\end{array}$ & 6,0 Dopamin & $\begin{array}{r}90 \\
2 \\
5\end{array}$ & $\begin{array}{l}86 \\
3\end{array}$ & $75-90$ \\
\hline
\end{tabular}


Diese Wiederfindungen stimmen mit den Literaturangaben überein $(3,21)$. Die Präzision der Wiederfindungsversuche wurde aus Fünffachbestimmungen in einer Serie bestimmt (Tabelle 2). Dabei waren die Aufstockzusätze so gewählt, daß sie dem Gehalt der nicht aufgestockten Probe entsprachen. Eine mögliche $\mathrm{Zu}-$ nahme der Streuung mit höherer Konzentration kann aus diesen Werten nicht abgeleitet werden.

\section{Nacbiveisgrenze}

Die aus Mittelwert plus Standardabweichung $(\overline{\mathbf{x}}+3 \mathrm{~s})$ der Leerwerte bestimmte Nachweisgrenze (22) liegt beim Adrenalin bei $0,5 \mu \mathrm{g} / \mathrm{l}$, bcim Noradrenalin bei $3 \mu \mathrm{g} / \mathrm{l}$ und beim Dopamin bei $10 \mu \mathrm{g} / l(19,20)$.

\section{Störeinfliisse}

Die Dopaminbestimmung wird selbst durch hohe Adrenalin- und Noradrenalinmengen nicht gestört (19). Dagegen wurde eine mehr oder minder starke wechselseitige Beeinflussung von Adrenalin und Noradrenalin und eine Störung durch Dopamin bei ihrer Bestimmung beobachtet. Der Störeinfluß durch Medikamente wurde an anderer Stelle besprochen (20).

Die Fluoreszenz des Noradrenalins beträgt etwa 5 Prozent der des Adrenalins bei gleicher Konzentration und Oxydation bei pH 2,8 $(1,2,20)$. Bei gesunden Probanden beträgt die Adrenalinausscheidung etwa 10 bis 20 Prozent der des Noradrenalins, so daß die Adrenalinwerte ohne Berücksichtigung der Störeinflüsse zu hoch bestimmt werden. Die Fluoreszenz einer gleich konzentrierten Dopaminlösung beträgt etwa 1 Prozent der des Noradrenalins nach Oxydation bei pH 6,0. Bei der Bestimmung der Normalwerte wurden in dem gleichen Eluat Dopamin und Noradrenalin bestimmt, so daß eine Korrektur der Noradrenalinwerte über einen mitgeführten Dopaminstandard möglich war. Die nicht korrigierten Werte liegen $8 \pm 3 \%(\bar{x} \pm s, n=98)$ höher als die korrigierten. Diese Korrektur wurde bei der Angabe des Normbereiches der Noradrenalinausscheidung aus methodischen Gründen nur in Klammern angegeben. Der zur Korrektur benutzte Dopaminstandard mußte nämlich fünffach höher konzentriert als bei der Dopaminbestimmung gewählt werden, um eine sicher meßbare Peakhöhe (Fluoreszenz) zu erhalten.

\section{Probeverwahrung}

Führt eine Person die Bestimmung der Katecholamine durch, so ist es rationeller, an einem Tag bei einer größeren Anzahl von Proben die Abtrennung und am nächsten Tag die Bestimmung vorzunehmen. Voraussetzung ist, $\mathrm{da} B$ in den Säuleneluaten keine Zerstörung der zu bestimmenden Substanz eintritt. Zur Überprüfung dieser Möglichkeit wurde folgender Versuch durchgeführt. Die Eluate von sechs verschiedenen Urinproben wurden aliquotiert. Ein Teil wurde am gleichen Tag analysiert (Tab 3, A), der restliche Teil bis zur Bestimmung nach etwa 24 Stdn bei $+4^{\circ}$ im Eisschrank aufbewahrt (Tab. 3, B). Die elektrometrisch bestimmten pH-Werte der Eluate lagen zwischen 3,2 und 3,4.
Tab. 3 Versuche zur Probeverwalirung mit 6 verscliedenen Poolurinproben.
$A \Rightarrow$ Eluate am gleichen Tag analysiert, $\mathrm{B}=$ Eluate nach 24stündiger Aufbewahrung bei $+4^{\circ} \mathrm{im}$ Eisschrank analysiert. Die angegebenen Werte sind Mittelwerte aus Dreifachbestinmungen

\begin{tabular}{ccccrrr}
\hline & \multicolumn{2}{c}{$\begin{array}{c}\text { Adrenalin } \\
\mu \mathrm{g} / 50 \mathrm{ml}\end{array}$} & \multicolumn{2}{c}{$\begin{array}{c}\text { Noradrenalin } \\
\mu \mathrm{g} / 50 \mathrm{ml}\end{array}$} & \multicolumn{2}{c}{$\begin{array}{c}\text { Dopamin } \\
\mu \mathrm{g} / 50 \mathrm{~m} /\end{array}$} \\
& A & B & A & B & \multicolumn{1}{c}{ A } & B \\
\hline 1 & 0,23 & 0,18 & 1,40 & 1,53 & 13,4 & 12,6 \\
2 & 0,54 & 0,54 & 2,29 & 2,19 & 4,4 & 3,1 \\
3 & 0,10 & 0,12 & 2,93 & 2,96 & 12,4 & 10,9 \\
4 & 0,24 & 0,23 & 2,96 & 3,08 & 7,4 & 7,7 \\
5 & 0,89 & 0,68 & 1,54 & 1,46 & 9,9 & 10,3 \\
6 & 0,33 & 0,35 & 2,73 & 3,00 & 4,6 & 5,1 \\
\hline
\end{tabular}

Nach diesen Versuchen (Tabelle 3) ist eine verlustfreie Verwahrung der Säuleneluate für alle drei Bestandteile unter den angegebenen Versuchsbedingungen möglich. Die Differenzen der sich jeweils entsprechenden Meßwerte liegen im Bereich der methodischen Streuung. Das Ergebnis der Probeverwahrung stimmt mit den Litcraturangaben überein $(17,23)$.

\section{Prüfung der Methode}

\section{Bestimmungsverfabren}

Für den untersuchten Konzentrationsbereich von $2 \mathrm{ng}$ bis $1 \mu \mathrm{g}$ Adrenalin/ml Probe und $5 \mathrm{ng}$ bis $1 \mu \mathrm{g}$ Noradrenalin $/ \mathrm{m} /$ Probe wurde eine lineare Abhängigkeit von Fluoreszenz und Konzentration gefunden.

Die Konzentrationen der einzelnen in der Arbeitsvorschrift angegebenen Reagenzien, die Einwirkungszeit des Oxydationsmittels und der alkalischen 2-Mercaptoäthanollösung wurden variiert. Diejenige Versuchsanordnung wurde als optimal angesehen, die die größte Fluoreszenz ergab. Das Ergebnis der Variation der Kupferionenkonzentration zeigte einige neue Gesichtspunkte, so daß darüber ausführlich berichtet werden soll.

Nach Weil-MaLHerbe beruht die Wirkung der Kupferionen auf einem katalytischen Effekt bei der Oxydation. Dies könnte aber nur eine Verkürzung der Oxydationszeit bedingen. Gegen einen katalytischen Effekt sprechen die Ergebnisse von HäGGENDAHL (10), daß nämlich die Fluoreszenzabnahme in alkalischer Lösung durch Kupferionen innerhalb eines gewissen Zeitabschnittes mit EDTA partiell aufgehoben werden kann. Dieser Versuch zeigt, daß eine Reaktion zwischen Fluorophor und Kupferionen stattfindet. Diese Annahme wird gestützt durch die Zunahme der Stabilität des Fluorophors bei Zugabe von Kupferionen (Abbildung 2). Die Fluoreszenzminderung bei Zugabe von Kupferionen nach der Oxydation zusammen mit der alkalischen 2-Mercaptoäthanollösung - eine Beobachtung $(1,2)$, die bestätigt werden kann - ist nicht beweisend für eine alleinige Wirkung des Kupfers bei der Oxydation. Dieser Effekt könnte darauf beruhen, daß die Kupferionen mit den SH-Gruppen des Reduktionsmittels reagieren und somit nicht mehr für eine Reaktion mit dem Fluorophor zur. Verfügung stehen. Nimmt man nämlich statt des 


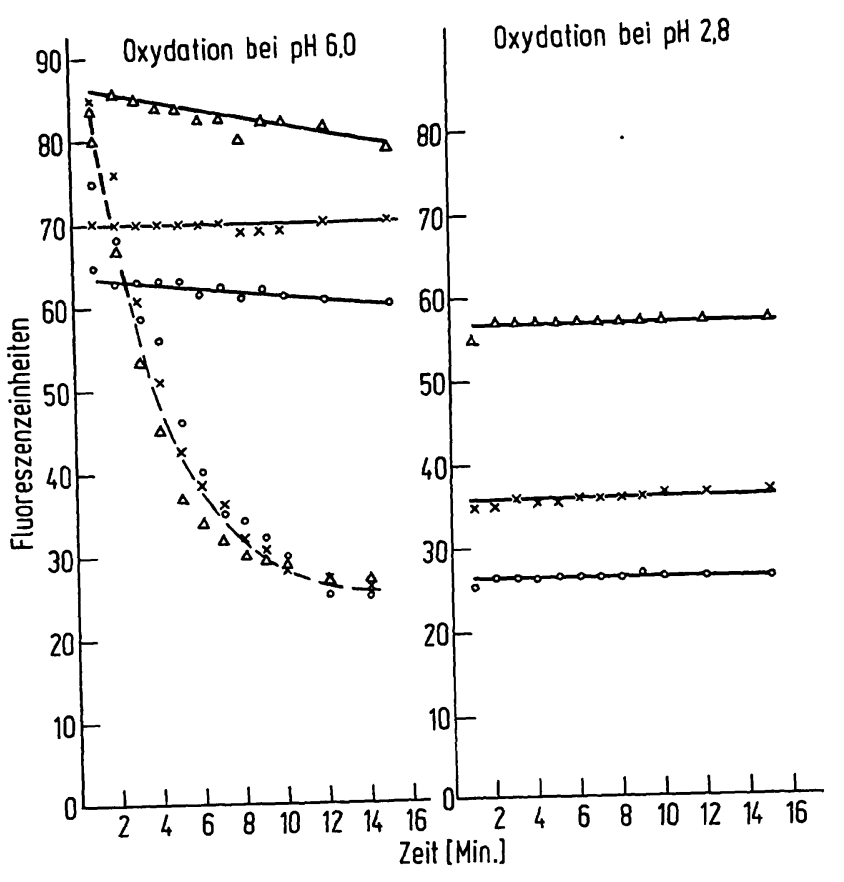

Abb. 2

Fluoreszenz in Abhängigkeit von der Zeit von drei verschiedenen Urinproben (ohne Kupferionenzusatz gestrichelte Linie)

SH-haltigen Reduktionsmittels Ascorbinsäure (Oxydation bei pH 6,0), so tritt dieser Effekt nicht ein. Dieses unterschiedliche Verhalten bei Verwendung von Ascorbinsäure als Reduktionsmittel ist schon bei HÄGGENDAHL (10) beschrieben worden.

Bei Untersuchungen über die Stabilität des Fluorophors wurden neben reinen Adrenalin- und Noradrenalinlösungen auch drei verschiedene Urinproben unterschiedlicher Adrenalin- und etwa gleicher Noradrenalinkonzentration untersucht. Das Ergebnis der Messung der Urinproben ist in Abbildung 2 wiedergegeben. Die Messungen wurden manuell durchgeführt. Die Volumina und Reaktionszeiten wurden analog der automatischen Bestimmung gewählt.

Für die Oxydation bei pH 6,0 wurden die Messungen mit und ohne Kupferacetatzusatz (0,01proz. Lösung) und für die Oxydation bei pH 2,8 nur mit Kupferionen in beiden Versuchsanordnungen ohne Ansäuern nach Thiolatzugabe - durchgeführt. Für die Oxydation bei pH 2,8 liegen die Meßpunkte einer Probe praktisch auf einer Geraden. Der Fluorophor ist für den untersuchten Zeitraum stabil. Hinsichtlich der Adrenalinkonzentration sind die Proben unterschiedlich. Für die Messung bei pH 6,0 lassen sich zwei Effekte erkennen. Einmal bewirkt der Zusatz der Kupferionen (durchgezogene Linie) eine Stabilisierung des Fluorophors. Zum anderen liegen die Meßpunkte für die drei verschiedenen Urinproben ohne Kupferionenżusatz eng beieinander (aus Übersichtsgründen nur eine gestrichelte Linie), während sie mit Kupferionen (durchgezogene Linie) voneinander getrennt liegen. Eine Erklärung für diesen Befund ergeben Versuche, die in Abbildung 3 zusammengefaßt sind. Hierbei wurde die Fluoreszenz gleicher Adrenalin-und Noradrenalinkonzentrationen bei $\mathrm{pH} 2,8$ und 6,0 mit unterschiedlicher Kupferionenkonzentration bestimmt.

Die Variation dieses Parameters. bei der Oxydation bei pH 2,8- allerdings hier nur in einem engen Konzentrationsbereich gemessen - zeigt einen geringen Einfluß auf die Fluoreszenz von Adrenalin und Noradrenalin. Ganz deutlich isst die Fluoreszenzzunahme des Adrenalins bei Zugabe der Kupferionen. Oxydiert man 'die Probe bei pH 6,0, so nimmt die Fluoreszenz des Noradrenalins mit steigender Kupferionenkonzentration zunächst etwas zu, dann wiederum $a b$, während die des Adrenalins kontinuierlich zunimmt. Nicht angeführt ist das Verhalten von Dopamin, dessen Fluoreszenz über den gemessenen Konzentrationsbereich praktisch unverändert niedrig blieb. Die in Abbildung 2 beobachteten Unterschiede der Fluoreszenz (Oxydation pH 6,0, mit Kupferionen) dürfen somit durch die Zunahme der Fluoreszenz von Adrenalin, dessen Konzentration in den drei Proben unterschiedlich ist, bedingt sein. Das unterschiedliche Verhalten von Adrenalin und Noradrenalin nach Oxydation bei pH 6,0 und steigender Kupferionenkonzentration bietet vielleicht eine Möglichkeit einer differentiellen Adrenalin- und Noradrenalinbestimmung, indem man die Proben ohne und mit Kupferionenzusatz bei einem schwach sauren $\mathrm{pH}$ oxydiert. Durch Variation des pH-Wertes um 6,0 und der Kupferionenkonzentration läßt sich möglicherweise eine Versuchsanordnung mit hoher Fluoreszenzausbeute für das Adrenalin und einer niedrigen für das Noradrenalin finden. Dieses Verfahren hätte den Vorzug, daß die Säuleneluate nicht

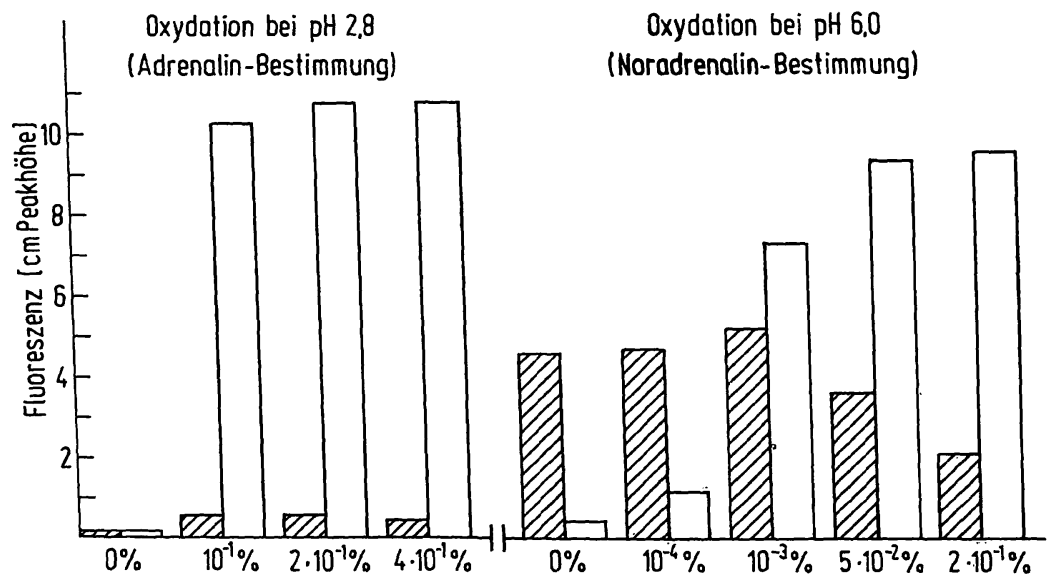

Abb. 3

Verhältnis der Fluoreszenz von Adrenalin (nicht schraffiert) und Noradrenalin (schraffiert) für unterschiedliche Kupferionenkonzentration und Oxydation bei pH 2,8 und 6,0. Auf der Abszisse sind die benutzten Kupferacetatmonohydratkonzentrationen in $\mathrm{g} / 100 \mathrm{ml}$ angegeben 
aliquotiert und auf unterschiedliche $\mathrm{pH}$-Werte eingestellt werden müßten.

Anlaß für das Einbeziehen von Noradrenalin in diese Untersuchung waren die Angaben, da $\beta$ Kupferionen in bestimmter Konzentration in alkalischer Lösung zu einer Fluoreszenzminderung führen (10), in schwach saurer oder neutraler Lösung die Fluoreszenz unbeeinflußt bleibt $(10,2)$. VALORI beobachtete eine Fluoreszenzsteigerung (18). Wie die Abbildung zeigt, ist die Fluoreszenz von Adrenalin und Noradrenalin in alkalischer Lösung von der Kupferionenkonzentration abhängig, wobei die optimalen Konzentrationen für Adrenalin ( $\mathrm{pH}$ 2,8) und Noradrenalin ( $\mathrm{pH} 6,0$ ) sich erheblich unterscheiden. Diese Konzentrationsabhängigkeit zeigt auch, daß eine Zunahme oder Abnahme der Fluoreszenz je nach Kupferionenkonzentration beobachtet werden kann.

Zusammenfassend kann gesagt werden, da $\beta$ die Ergebnisse der eigenen Versuche über den Einfluß der Kupferionen auf die Stabilität des Fluorophors sowie die Fluoreszenzintensität ebenfalls gegen einen einfachen katalytischen Effekt bei der Oxydation sprechen. Insbesondere die Stabilitätszunahme zeigt, daß eine Reaktion zwischen dem Fluorophor und den Kupferionen stattfindet. Allerdings sei eingeräumt, da $B$ auch nach diesen Versuchen noch keine eindeutige Aussage über den Reaktionsablauf gemacht werden kann. Ein Ansäuern der Probe nach Thiolatzugabe $(1,2)$ zur Stabilisierung des Fluorophors ist bei Anwesenheit von Kupferionen nicht erforderlich. Allerdings ist bei der Noradrenalinbestimmung $\mathrm{zu}$ berücksichtigen, daß je nach Kupferionenkonzentration die Fluoreszenzintensität des Adrenalins erheblich zunehmen kann, so daß sein Störeinfluß auf die Noradrenalinbestimmung nicht mehr vernachlässigt werden darf. Von Bedeutung ist diese Stabilisierung des Fluorophors allerdings nur bei manueller Durchführung der Bestimmung.

\section{Abtrennverfabren}

Zur Abtrennung der Katecholamine aus dem Urin sind verschiedene Verfahren beschrieben worden. Neben der Verwendung von Ionenaustauschern hat die Adsorption an Aluminiumoxid bei $\mathrm{pH}$ 8,3-8,5 breite Anwendung gefunden. Führt man die Elution in zwei Schritten durch, so erhält man zwei Fraktionen der Katecholamine und ihrer Metabolite. Bei Elution mit 0,2M Essigsäure werden zunächst die Katecholamine und ein kleiner Anteil der Katecholsäuren desorbiert. Gibt man anschließend 1N Schwefelsäure in die Säule, wird der Hauptteil det Katecholsäuren eluiert (24). Die erste Fraktion enthält Adrenalin, Noradrenalin, Dopamin und Dopa. Unter physiologischen Verhältnissen ist Dopa im Urin in nicht nachweisbaren Mengen vorhanden (3). Die hohe Umsatzgeschwindigkeit von Dopa bedingt, $\mathrm{da} ß$ es sich dem Nachweis entzieht (25).

Bei erhöhten Dopaminausscheidungen sollte man die von BertLer und Mitarbeiter (5) vorgeschlagene DopaDopamin-Trennung über einen Ionenaustauscher durch- führen. Die in Tabelle 7 angegebenen Dopaminwerte wurden ohne diese 'Trennung ermittelt.

Die Adsorption an Aluminiumoxid wird mit verschiedenen Techniken durchgeführt. Dabei kommen zur Anwendung Säulen- und batch-Technik $(1,21)$, sowie eine Kombination der beiden Verfahren (3). Verwendet man an Stelle des von CROUT (3) empfohlenen Magnetrührers bei der Adsorption einen mechanischen Rührer, dann entfällt der Einwand (24), daß bei diesem Vorgehen beim Einstellen des $\mathrm{pH}$-Wertes und dem nachfolgenden Rühren das Aluminiumoxid zerkleinert wird und durch die Säule läuft. Bei den im folgenden beschriebenen Versuchen wurde der Einfluß folgender Parameter auf die Wiederauffindung geprüft, die $\mathrm{pH}$-Abhängigkeit der Adsorption, der Einfluß der Rührzeit nach Einstellen des $\mathrm{pH}$-Wertes, die Menge des eingesetzten Aluminiumoxides und die unterschiedliche Abtrenntechnik.

Abbängigkeit und Einfluß der Rübrzeit auf die Adsorption Das pH-Optimum für die Adsorption der Katecholamine an Aluminiumoxid liegt nach den Ergebnissen der meisten Untersucher bei pH 8,3-8,5. Nach CHANG (26) soll die Adsorption der Katecholamine schon bei pH 7,0 vollständig sein. Crout (3) empfiehlt bei seiner Abtrenntechnik die Probe nach Einstellen des $\mathrm{pH}$-Wertes auf

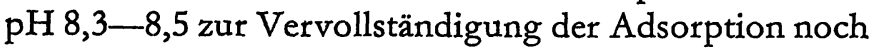
7 Min. zu rühren. Der Einfluß der beiden Parameter wurde nachuntersucht (Tabelle 4).

Tab. 4

Einfluß des pH-Wertes der Urinproben bei 10 minütigem Rühren nach Erreichen des $\mathrm{pH}$-Wertes und der Rührzeit nach Einstellen der Proben auf $\mathrm{pH} 8,5$ auf die Adsorption der Katecholamine an Aluminiumoxid.
Die angegebenen Werte sind Mittelwerte aus Dreifachbestimmungen. $A=$ fluorometrische Bestimmung $B=$ radiochemische Bestimmung.

$A=$ Adrenalin, $\mathrm{NA}=$ Noradrenalin, $\mathrm{DM}=$ Dopamin

\begin{tabular}{|c|c|c|c|c|c|c|c|}
\hline \multirow[t]{2}{*}{$\mathrm{pH}$} & \multirow[b]{2}{*}{ A } & \multicolumn{2}{|c|}{$\underset{\mathrm{A}}{\text { Ausbeute }} \%$} & \multicolumn{2}{|c|}{$\begin{array}{l}\text { Rührzeit } \\
\text { (Min.) }\end{array}$} & \multicolumn{2}{|c|}{$\underset{A}{\text { Ausbeute }}{ }_{B} \%$} \\
\hline & & 100 & 100 & & A & 89 & 92 \\
\hline \multirow[t]{2}{*}{8,5} & NA & 100 & 100 & 0 & NA & 93 & 89 \\
\hline & DM & 100 & 100 & & DM & 95 & 97 \\
\hline \multirow{3}{*}{7,0} & A & 39 & 56 & & A & 104 & 99 \\
\hline & NA & 37 & 54 & 5 & NA & 94 & 102 \\
\hline & DM & 34 & 54 & & DM & 95 & 100 \\
\hline \multirow{3}{*}{6,0} & A & 4 & 2 & & A & 100 & 100 \\
\hline & NA & 3 & 2 & 10 & NA & 100 & 100 \\
\hline & $\mathrm{DM}$ & 3 & 6 & & $\mathrm{DM}$ & 100 & 100 \\
\hline
\end{tabular}

Dazu wurden Adrenalin, Noradrenalin und Dopamin in $40 \mathrm{~m} l$ Poolurin bei verschiedenem $\mathrm{pH}$-Wert und konstanter Rührzeit von $10 \mathrm{Min}$. bzw. bei unterschiedlicher Rührzeit und konstantem pH-Wert von 8,5 bei der Adsorption fluorometrisch bestimmt (Tab. 4, A). Die fluorometrischen Werte wurden durch radiochemische Bestimmung der Wiederfindung überprüft (Tab. 4, B). Die Werte für die Versuchsbedingung, die die höchsten Ausbeuten ergab, also die Adsorption bei $\mathrm{pH} 8,5$ und 10 Min. Rührzeit, wurden 100 Prozent gesetzt und die übrigen Werte in Prozent dieser Werte umgerechnet. Die Ergebnisse hinsichtlich des Einflusses der Rührzeit sind eine Bestätigung für das von Crout (3) cmpfohlene Vor- 
gehen. Die pH-Abhängigkeit stimmt mit den Angaben der meisten Untersucher überein, nicht aber mit denen von Chang (26).

Einfluß der Menge an Aluminiumoxid und der Abtrenntechnik auf die Adsorption

Wie in der Arbeitśvorschrift angegeben, wird die zur Analyse eingesetzte Urinmenge nach Zusatz von Aluminiumoxid mit demin. Wasser auf etwa $180 \mathrm{ml}$ aufgefüllt. Anschließend wird der $\mathrm{pH}$-Wert eingestellt. Für unterschiedliche Mengen an Aluminiumoxid wurden die Wiederfindungsraten für Adrenalin, Noradrenalin und Dopamin bestimmt (Tabelle 5). Die in Klammern angegebenen Werte wurden fluorometrisch ermittelt. Außerdem wurde die Wiederfindung mit der Säulentechnik bestimmt.

Tab. 5

Wiederfindung in Abhängigkeit von der eingesetzten Aluminiumoxidmenge und der Technik des Abtrennverfahrens. Die angegebenen menge unde sind Mittelwerte aus Dreifachbestimmungen

\begin{tabular}{|c|c|c|c|c|}
\hline \multirow[t]{2}{*}{ Substanz } & \multicolumn{3}{|c|}{$\begin{array}{c}\text { Adsorption nach CROUT } \\
\text { (Ausbeute \%) }\end{array}$} & \multirow{2}{*}{$\begin{array}{c}\text { Chromato- } \\
\text { graphie } \\
\text { (Ausbeute \%) } \\
2 \mathrm{~g} \mathrm{Al}_{2} \mathrm{O}_{3}\end{array}$} \\
\hline & $1 \mathrm{~g} \mathrm{Al}_{2} \mathrm{O}_{3}$ & $2 \mathrm{~g} \mathrm{Al}_{2} \mathrm{O}_{3}$ & $3 \mathrm{~g} \mathrm{Al}_{2} \mathrm{O}_{3}$ & \\
\hline Adrenalin & 74 & 81 & $\begin{array}{c}82 \\
(86)\end{array}$ & $\begin{array}{c}77 \\
(78)\end{array}$ \\
\hline Noradrenalin & 75 & 84 & $\begin{array}{c}84 \\
(94)\end{array}$ & $\begin{array}{c}79 \\
(84)\end{array}$ \\
\hline Dopamin & 73 & 82 & 85 & 78 \\
\hline
\end{tabular}

Für die benutzte Art der Abtrennung besteht zwischen $2 \mathrm{~g}$ und $3 \mathrm{~g}$ Aluminiumoxid kein Unterschied in den Wiederfindungsraten. Bei Verwendung von $1 \mathrm{~g}$ liegen die Ausbeuten niedriger. Die Wiederfindung mit der Säulentechnik, die mit den gleichen Volumina wie die kombinierte Abtrenntechnik durchgeführt wurde, ist bei Verwendung gleicher Aluminiumoxidmengen niedriger als mit der Technik von Crout. Dies ist nicht als Beweis für die Überlegenheit der letzteren über die Säulentechnik anzusehen; denn es ist durchaus möglich, daß bei Variation der Volumina und der Aluminiumoxidmenge mit der Säulentechnik die gleichen Ausbeuten erreicht werden. Andererseits besteht aber kein Grund zu der Annahme, daß zwischen beiden Verfahren grundsätzliche Unterschiede bestehen.

\section{Vorbebandlung des Aluminiumoxids}

Von verschiedenen Autoren wird vorgeschlagen, das Aluminiumoxid vor Benutzung mit Säure zu waschen, um Spuren von Schwermetallen, Alkali und ganz feine Partikel zu entfernen $(2,3)$. Der Einfluß der Vorbehandlung wurde an reinen Lösungen und Poolurinproben geprüft, indem nicht vorbehandeltes, mit demin. Wasser und mit $2 \mathrm{~N}$ Salzsäure vorbehandeltes Aluminiumoxid-Woelm für die Abtrennung der Katecholamine benutzt wurde. Die. Vorbehandlung des Aluminiumoxides wurde analog der Vorschrift von CROUT (3) durchgeführt. Die Bestimmung der Ausbeute mit unterschiedlich vorbehandeltem Aluminiumoxid erfolgte radiochemisch und fluorometrisch (Tabelle 6 ).

Nach diesen Messungen besteht also ein eindeutiger Unterschied $z$ wischen unbehandeltem und vorbehan-
Tab. 6

Vergleich der Wiederfindungen mit verschieden vorbehandeltem Aluminiumoxid. Die mit säuregewaschenem Aluminiumoxid ermittelten Werte wurden für jeden Bestandteil gesondert $100 \%$ gesetzt und die Werte der beiden anderen Versuchsreihen in Prozent dieser Werte umgerechnet. Die angegebenen Meßwerte sind Mittelwerte aus Dreifachbestimmungen

$A=$ fluorometrische,$B=$ radiochemische Bestimmung

\begin{tabular}{|c|c|c|c|c|c|c|c|}
\hline \multirow[b]{3}{*}{ Probe } & \multirow[b]{3}{*}{ Substanz } & \multicolumn{6}{|c|}{$\begin{array}{c}\mathrm{Al}_{2} \mathrm{O}_{\mathrm{g}} \\
\text { (Ausbeute in \%) }\end{array}$} \\
\hline & & \multirow{2}{*}{\multicolumn{2}{|c|}{$\underset{A}{\text { nicht }} \underset{\text { vorbehandelt }}{\text { B }}$}} & \multicolumn{4}{|c|}{ vorbehandelt mit } \\
\hline & & & & & Ber & A & $\mathrm{re}_{\mathrm{B}}$ \\
\hline $\begin{array}{l}\text { reine } \\
\text { Lösung }\end{array}$ & $\begin{array}{l}\text { Adrenalin } \\
\text { Noradrenalin } \\
\text { Dopamin }\end{array}$ & $\begin{array}{l}89 \\
79 \\
80\end{array}$ & $\begin{array}{l}88 \\
89 \\
77\end{array}$ & $\begin{array}{r}100 \\
96 \\
99\end{array}$ & $\begin{array}{l}98 \\
99 \\
90\end{array}$ & $\begin{array}{l}100 \\
100 \\
100\end{array}$ & $\begin{array}{l}100 \\
100 \\
100\end{array}$ \\
\hline $\begin{array}{l}40 \mathrm{ml} \\
\text { Poolurin }\end{array}$ & $\begin{array}{l}\text { Adrenalin } \\
\text { Noradrenalin } \\
\text { Dopamin }\end{array}$ & $\begin{array}{r}100 \\
84 \\
87\end{array}$ & $\begin{array}{l}90 \\
95 \\
88\end{array}$ & $\begin{array}{r}100 \\
96 \\
90\end{array}$ & $\begin{array}{l}97 \\
97 \\
98\end{array}$ & $\begin{array}{l}100 \\
100 \\
100\end{array}$ & $\begin{array}{l}100 \\
100 \\
100\end{array}$ \\
\hline
\end{tabular}

deltem Aluminiumoxid. Für den ersteren Fall liegen die Ausbeuten eindeutig niedriger. Ob ein wirklicher Unterschied zwischen wasser- und säuregewaschenem Aluminiumoxid besteht, müßte durch eine größere Anzahl von Messungen geprüft werden. $\mathrm{Da}$ aber der zusätzliche Arbeitsaufwand für die Vorbehandlung mit Säure gering ist, ist diese Fragestellung ohne praktische Bedeutung. Bei allen Versuchen wurde daher säuregewaschenes Aluminiumoxid benutzt. Für die eigenen Arbeiten wurde die Regelung eingeführt, daß für jede neue Charge Aluminiumoxid die Wiederfindung radiochemisch mit ${ }^{14} \mathrm{C}$-markiertem Adrenalin überprüft wird.

\section{Elutionsprofil der adsorbierten Katecholamine}

Bei dem Verfahren von Crour werden nach dem Waschen der Säule $\left(3 \mathrm{~g} \mathrm{Al}_{2} \mathrm{O}_{3}\right)$ mit demin. Wasser $3 \mathrm{ml} 0,2 \mathrm{~N}$ Essigsäure auf die Säule gegeben. Diese Vorfraktion wird verworfen, die eigentliche Elution anschließend mit $8 \mathrm{ml} 0,2 \mathrm{M}$ Essigsäure durchgeführt. Es sollte untersucht werden, ob mit der Vorfraktion nicht schon Katecholamine miteluiert werden und welchen Einfluß das "Vorwaschen" auf das Elutionsprofil hat. Dies wurde in getrennten Versuchen mit markiertem Adrenalin, Noradrenalin und Dopamin geprüft.

Es wurden $40 \mathrm{~m} l$ Poolurin mit den entsprechenden markierten Verbindungen aufgestockt, Vorfraktion und Eluat in $1 \mathrm{~m} l$ Fraktionen gesammelt und deren Aktivität (gestrichelte Linie) bestimmt. In einer zweiten Versuchsreihe wurde die Vorelution mit $3 \mathrm{ml} 0,2 \mathrm{M}$ Essigsäure weggelassen. Sofort nach dem Waschen mit demin. Wasser wurden $8 \mathrm{~m} l 0,2 \mathrm{M}$ Essigsäure auf die Säule gegeben (durchgezogene Linie). Beide Versuche wurden mit $2 \mathrm{~g}$ und $3 \mathrm{~g}$ Aluminiumoxid durchgeführt.

In Abbildung 4 ist das Ergebnis dieser Versuchsreihen am Beispiel des Adrenalins für unterschiedliche $\mathrm{Al}_{2} \mathrm{O}_{3}$ Mengen wiedergegeben. Die Vorfraktionen sind nicht mit eingezeichnet. Ihre Aktivitäten lagen in der Größenordnung des Nulleffekts. Nimmt man die Vorfraktionen dazu, dann decken sich die beiden Elutionsprofile. Für Noradrenalin und Dopamin liegen die Elutionsmaxima in den gleichen Fraktionen. Ihre Lage ist einmal von der Menge des eingesetzten Aluminiumoxids abhängig und zum anderen von der Vorelution mit Essigsäure. Wie man aus Abbildung 4 ersieht, ist das Elutionsprofil nicht 


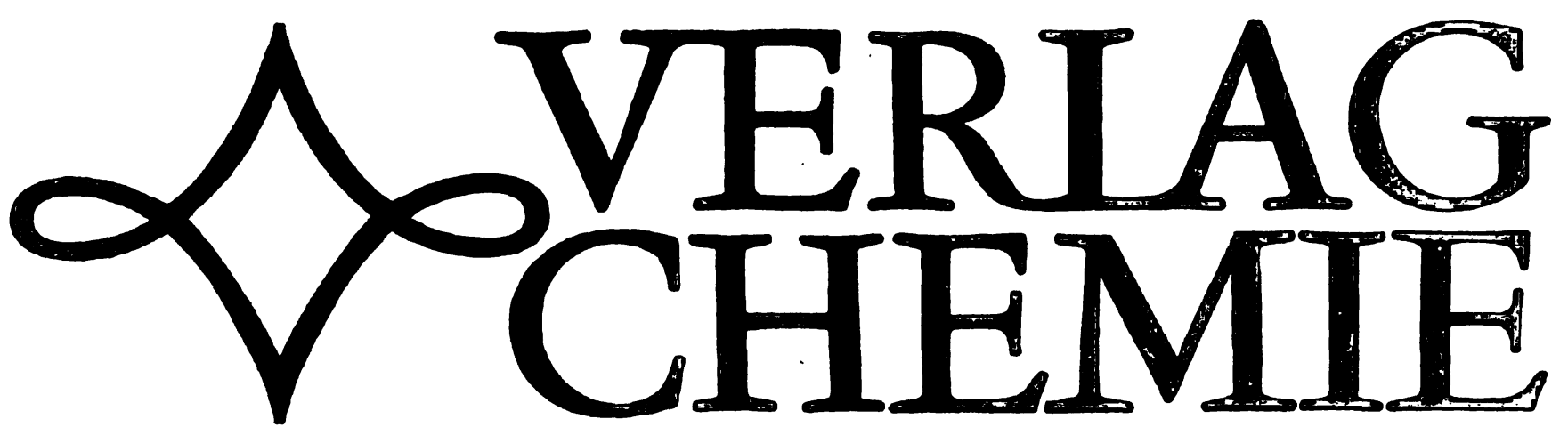

\section{Hellmut Fiedler}

\section{Chemisches Rechnen}

\section{auf elementarer Grundlage in Form einer Aufgabensammlung}

1970. 7. verbesserte Auflage. 368 Seiten mit 28 Abbildungen. Broschiert DM 19,80.

(Lizenzausgabe für die Bundesrepublik Deutschland.)

Der Anfänger hat gewöhnlich Mühe mit der Berechnung chemischer Aufgaben, und er gerät in Schwierigkeiten, wenn er den vermeintlich so einfachen Dreisatz oder die Verhältnisgleichung auf komplizierte Mischungsprobleme anwenden soll. Auch erfordert es erfahrungsgemä $ß$ meist einige Ubung, bis mit chemischen Formeln ein sicheres Gefühl für die durch sie symbolisierten Stoffmengen verbunden wird.

Dieses Buch führt mit kurzen Erläuterungen in die verschiedenen Gebiete des chemischen Rechnens ein: Mischungsvorgänge, Herstellung von Lösungen bestimmter Konzentration, Berechnung der prozentualen Zusammensetzung von Verbindungen, Ermittlung von stöchiometrischen Faktoren, Anwendung des idealen Gasgesetzes, quantitative Analyse; und es gibt anhand von über 400 Aufgaben Gelegenheit, sich die notwendige Beherrschung des Stoffes anzueignen. Die 400 in den Text eingebauten Aufgaben werden mit ausführlichen Lösungsgängen bis zum Endresultat entwidkelt und setzen den Leser in die Lage, die restlichen Aufgaben selbständig zu lösen. Der Vergleich mit den im Anhang zusammengestellten Resultaten bietet eine Kontrolle, ob der erwünschte Lernerfolg eingetreten ist.

Im Anhang finden sich außerdem noch tabellarische Zusammenstellungen von Atom- und Molekularmassen und ihren Logarithmen, Dichtetabellen und eine fünfstellige Logarithmentafel.

\section{VERLAG CHEMIE - GMBH WEINHEIM/BERGSTR.}




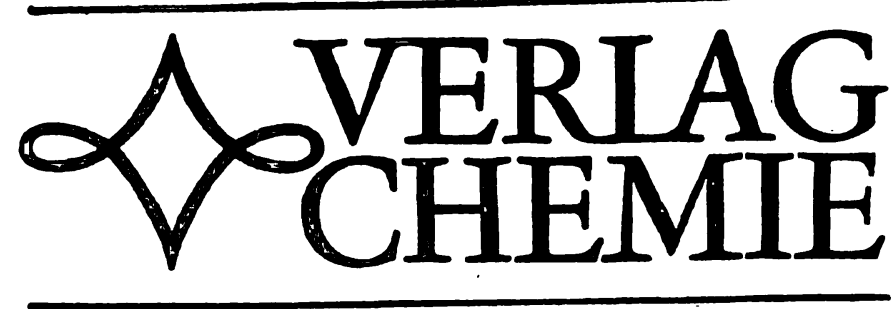

Soeben erscbeint in neuer Auflage:

\section{Klinisches Labor}

1970. 11. Auflage der Medizinisch-cbemischen Untersucbungsmetboden. 632 Seiten mit zablreichen Abbildungen und Tabellen, einer umfangreichen Normalwertetabelle, einem Literaturverzeicbnis zur Normalwertetabelle sowie einem Reagenzien- und Sachverzeichnis. Kunststoffeinband DM 20,-

(Eine V'eröffentlichung der E. Merck AG., Darmstadt)

U'ber dieses Bucb:

Die rasche Entwicklung der klinischen Laboratoriumsdiagnostik machte eine gründliche Uberarbeitung und umfangreiche Erweirerung dieser Methodensammlung erforderlich. Dem Inhalt der 11. Auflage liegt eine Umfrage der Herausgeber bei klinischen Chemikern und Laboratoriumsärzten zugrunde, um eine möglichst praxisnahe Auswahl moderner Labormethoden zu treffen. Die Bearbeitung der klinisch-chemischen Methoden übernahm Prof. Dr. R. Clotten, Freiburg, die der flammenphotometrischen Serumanalyse Prof. Dr. R. Herrmann, Gießen; das Kapitel über Mikrolitermethoden schrieb Prof. Dr. H. Mattenheimer, Chicago. Bei der Abfassung des Abschnittes über die Prüfung des Kohlenhydratstoffwechsels wirkte Prof. Dr. H. Mehnert, München, maßgeblich mit. Die hämatologischen Methoden wurden von Prof. Dr. W. Hunstein, Göttingen, und Dr. H.-G. Harwerth, Freiburg, bearbeitet.

$\mathrm{Da}$ die Arbeiten zur Standardisierung der Labormethoden noch im Gange sind und in verschiedenen Laboratorien teilweise unterschiedliche Methoden gebräuchlich sind, wurden bei wichtigen Bestimmungen und Nachweisen mehrere Techniken dargestellt, um dem Benutzer die Möglichkeit zu geben, das für seine Zwecke am besten geeignete Verfahren zu wählen. Bei Methoden, mit denen keine eigenen Erfahrungen vorlagen, werden Hinweise auf die Originalliteratur gegeben, die der an der Durchführung Interessierte zu Rate ziehen kann. Neu aufgenommen wurde eine ausführliche Normalwertetabelle, deren Daten durch Literaturzitate belegt sind. Nach Möglichkeit ist dort auch die Methodik genannt, mit der die Normalwerte ermittelt worden sind.

Wir iibersenden Ibnen gern unseren Sonderprospekt.

VERLAG CHEMIE - GMBH WEINHEIM/BERGSTR.
Holleman - Wiberg

\section{Lehrbuch}

\section{der anorganischen Chemie}

Begründet von A. F: HollemanN $\dagger$

. 1

Von Dr. Dr. h. c. Dr. h. c. Egon Wrberg, Professor an der Universität München

71. - 80., völlig umgearbeitete und stark

erweiterte Auflage

mit einem Anhang Chemiegeschichte, Raumbilder-Erläuterungen, einem Tabellen-Anhang, sowie 216 Figuren und einer Beilage von 37 Struktur-Bildern in stereoskopischer Darstellung.

Groß-Oktav. XXXII, 1209 Seiten. 1971.

Werkstoff DM 58,-

Der Text der 71.-80. Auflage des Lehrbuches wurde völlig umgestaltet und stark erweitert, so $\mathrm{da} \beta$ ein neues Werk entstanden ist, das sich jetzt nicht mehr - wie bisher, nur an den Anfänger, sondern auch an die Fortgeschrittenen der Chemie wendet.

Das Buch gliedert sich in vier große Hauptteile:

A: Atom und Molekül

B: Hauptgruppen des Periodensystems

C: Nebengruppen des Periodensystems

D: Lanthaniden und Actiniden

Den Abschluß des Buches bilden: ein chemiegeschichtlicher Anhang, ein Anhang mit Erläuterungen zur angefügten Raumbilder-Beilage und ein Tabellen-Anhang. Die Atomgewichte, Elementhäufigkeiten, physikalische Daten und atomaren Konstanten entsprechen dem neuesten Stand.

Die Anzahl der Abbildungen, Tabellen und tabellarischen Úberblicke wurde beträchtlich erhöht. Die Raumbilder-Beilage wurde um 6 Atomstrukturen vermehrt.

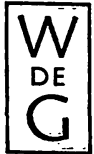

Walter de Gruyter \& Co • Berlin 30 


\section{Abb. 4}

Elutionsprofil von Adrenalin nach Adsorption an Aluminiumoxid mit Vorelution (gestrichelte Linie) und ohne Vorelution (durchgezogene Linie)

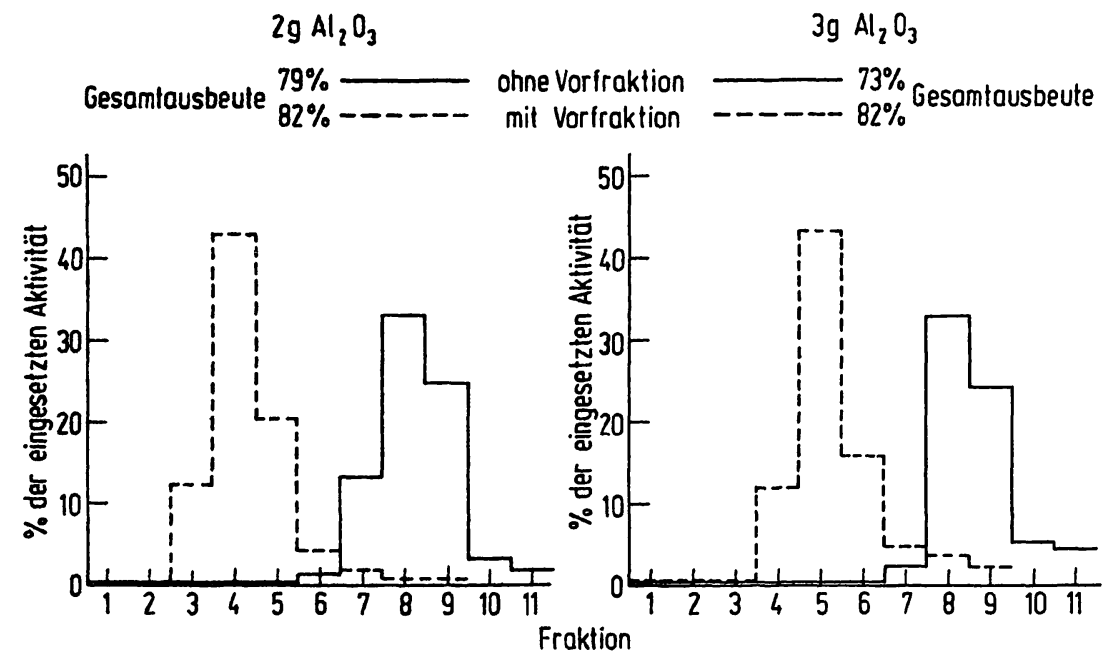

scharf, sondern es „tailt". Je eher die Elution einsetzt, desto mehr wird noch von der zu bestimmenden Substanz miterfaßt.

\section{Anwendung der Methode}

Das beschriebene Verfahren wurde bei der Analyse von einigen 3-Stunden-Urinproben aus einer Versuchsreihe über den Einfluß von Licht-Dunkel-Wechsel als Zeitgeber für circadiane Rhythmen (27) und zur Bestimmung der Ausscheidung der Katecholamine im 24Stunden-Urin angewandt. Außerdem wurde die Ausscheidung der Katecholamine und der Vanillinmandelsäure bei einem operativ gesicherten Phäochromocytom bestimmt.

\section{Tagesgang}

In Abbildung 5 ist das Ergebnis der Analyse von 3-Stunden-Urinproben von einer Versuchsperson wiedergegeben, und zwar für Adrenalin (unten), Noradrenalin (Mitte) und Dopamin (oben).

Bei diesem Versuch durfte die Versuchsperson während der beiden ersten Versuchstage nachts durchschlafen. In den beiden darauffolgenden Tagen wurde sie nachts in dreistündigem Abstand geweckt. Auffallend ist die höhere Dopaminausscheidung in den Nachtstunden während der beiden letzten Versuchstage.

\section{Normalverte}

Die 24-Stunden-Urinproben stammen von einer Stichprobe von 47 gesunden Frauen und 51 Männern im Alter zwischen 18 und $65 \mathrm{Jahren}$, die ihren normalen Beschäftigungen nachgingen. Die Einnahme von Medikamenten war drei Tage vor Sammelbeginn untersagt, ebenso der Verzehr von Schokolade, vanillehaltigen Speisen, Tonic Water und Bananen. Rauchen und mäBiges Trinken von Alkohol waren erlaubt. GrEEN und WALKER (28) haben allerdings jüngst in einer Kurzmitteilung dargelegt, daß diätetisch zugeführtes Vanillin die Vanillinmandelsäure-Bestimmung (Oxydation mit Natrium-meta-perjodat) nicht stört. Während des Sammelns wurden die Proben kühl aufbewahrt. Nach Be-
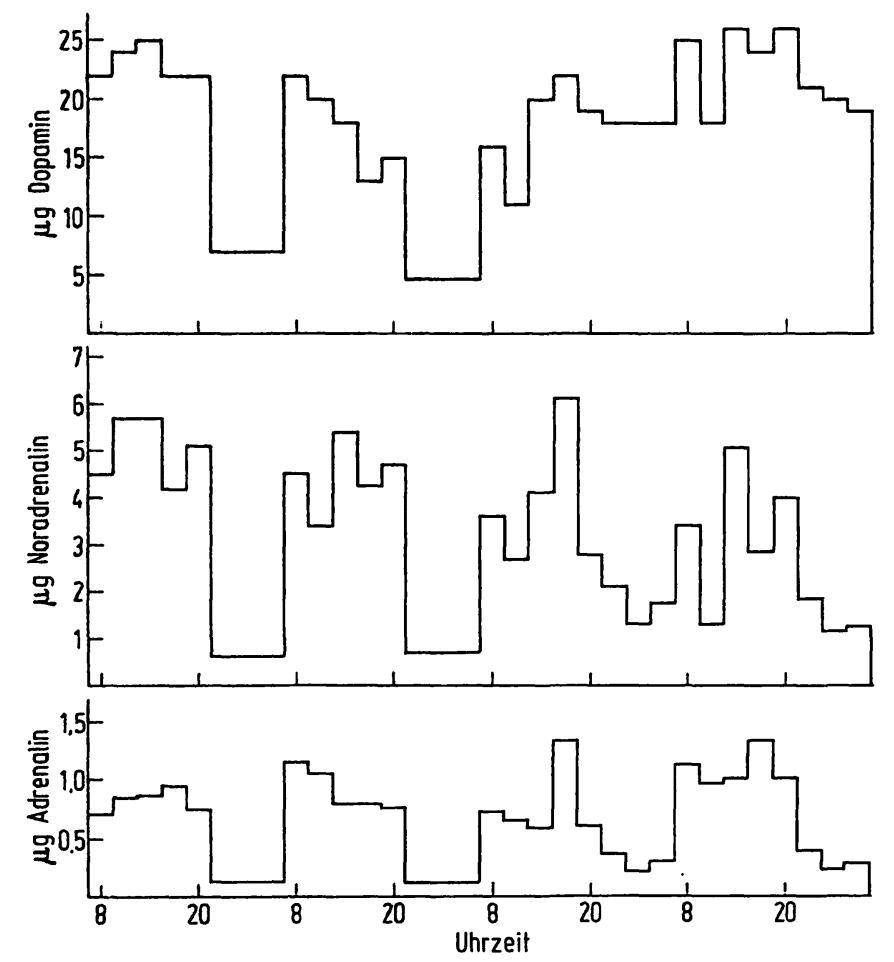

Abb. 5

Tageszeitliche Änderung der Adrenalin-, Noradrenalin- und Dopaminausscheidung bei einer Versuchsperson an 4 aufeinanderfolgenden Versuchstagen

endigung der Sammelperiode wurden die Urine mit Salzsäure ( $10 \mathrm{~m} l 6 \mathrm{~N} \mathrm{HCl} / l$ Urin) angesäuert und bis zur Analyse bei $-28^{\circ}$ in einer Tiefkühltruhe aufbewahrt.

Wie eingangs erwähnt, wurden in dem gleichen auf pH 6,0 eingestellten Eluat Noradrenalin, Gesamtkatecholamine und Dopamin bestimmt. Außerdem wurde in einem auf $\mathrm{pH}$ 2,8 eingestellten aliquotierten Teil der Probe Adrenalin bestimmt.

Die Ausscheidung der genannten Verbindungen im 24-Stunden-Urin des Normalkollektivs - nicht korrigiert auf eine mittlere Wiederfindung von 80 Prozent ist in Abbildung 6 in Abhängigkeit vom Alter wiedergegeben. In einer vorhergehenden Arbeit wurden die Werte der Vanillinmandelsäure-Ausscheidung in den gleichen 24-Stunden-Urinproben veröffentlicht (29). 
Die Vanillinmandelsäureausscheidung war nicht altersabhängig. Wie Abbildung 6 zeigt, liegt auch keine Altersabhängigkeit der Ausscheidung von Adrenalin, Noradrenalin und Dopamin vor.

Der Normalbereich der 24-Stunden-Ausscheidung ist in Tabelle 7 zusammengefaßt.

Auffällig ist, daß der Median für alle Bestandteile bei den Frauen niedriger liegt als bei den Männern. Dies gilt auch für die Vanillinmandelsäureausscheidung (29). Allerdings ist der Lageunterschied nur für die Adrenalinund Dopaminausscheidung statistisch signifikant (Wilcoxon-Test, $\mathrm{P}=0.01$ ). $\mathrm{Da}$ aber bei allen untersuchten Bestandteilen der Median für die weibliche Stichprobe niedriger ist als für die männliche, ist die Vermutung berechtigt, daß - auch bei nicht statistischer Signifikanz - die Ausscheidung der Frauen statistisch gesehen doch niedriger ist. $\mathrm{Da}$ die Differenzen im Vergleich zur biologischen Streuung zum Teil sehr gering sind, kann man vermuten, daß bei einer größeren Stichprobe dieser Befund für alle Bestandteile statistisch zu sichern wäre.

Erwähnt sei, daß in der von KäRkÏ (23) mit einer biologischen Methode gewonnenen Stichprobe in der gleichen Altersgruppe Frauen ebenfalls eine statistisch signifikant niedrigere Adrenalinausscheidung haben als Männer, während die Differenz bei der Noradrenalinausscheidung nicht statistisch signifikant.ist (gleicher Test, gleiches Testniveau).
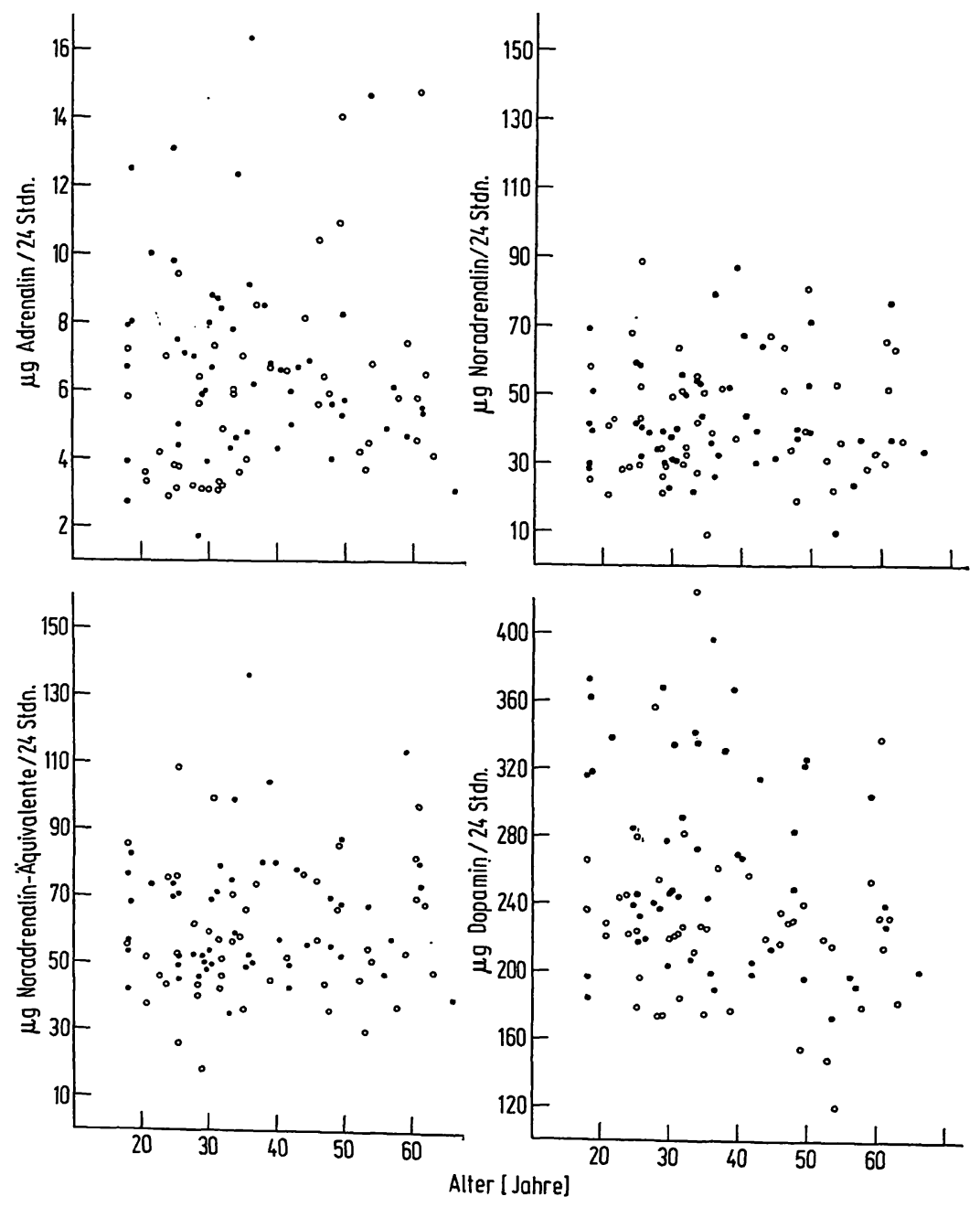

Abb. 6

Ausscheidung von Adrenalin, Noradrenalin, Gesamtkatecholaminen und Dopamin im 24-StundenUrin bei 47 Frauen (Kreise) und 51 Männern (Punkte) in Abhängigkeit vom Alter

Tab. 7

Normalbereich der Ausscheidung von Adrenalin, Noradrenalin, Gesamtkatecholaminen und Dopamin im 24-Stunden-Urin. Die in Klammern angegebenen Werte sind hinsichtlich des Dopaminstöreinflusses korrigiert

\begin{tabular}{|c|c|c|c|c|c|c|}
\hline \multirow[b]{2}{*}{ Bestandteil } & \multicolumn{2}{|c|}{. Männer $\mathrm{n}=51$} & \multicolumn{2}{|c|}{ Frauen $n=47$} & \multicolumn{2}{|c|}{ Gesamt $n=98$} \\
\hline & $\begin{array}{c}\text { Median } \\
(\mu \mathrm{g})\end{array}$ & $\begin{array}{c}\text { 96-Perzentil } \\
(\mu \mathrm{g})\end{array}$ & $\begin{array}{c}\text { Median } \\
(\mu \mathrm{g})\end{array}$ & $\begin{array}{c}\text { 96-Perzentil } \\
(\mu \mathrm{g})\end{array}$ & $\begin{array}{l}\text { Median } \\
(\mu \mathrm{g})\end{array}$ & $\begin{array}{c}\text { 96-Perzentil } \\
(\mu \mathrm{g})\end{array}$ \\
\hline Adrenalin & 6.6 & 2,7 & & 2,9 & & $2, \overline{7}$ \\
\hline & 0,0 & 14,6 & 4,6 & 9,4 & 5,8 & 14,6 \\
\hline Noradrenalin & $\begin{array}{c}40 \\
(36)\end{array}$ & $\begin{array}{l}22(19) \\
88(75)\end{array}$ & $\begin{array}{c}37 \\
(34)\end{array}$ & $\begin{array}{l}19(16) \\
81(77)\end{array}$ & $\begin{array}{c}39 \\
(36)\end{array}$ & $\begin{array}{l}19(16) \\
88(83)\end{array}$ \\
\hline $\begin{array}{l}\text { Gesamt- } \\
\text { Katecholamine }\end{array}$ & 58 & $\begin{array}{r}40 \\
114 \\
\end{array}$ & 56 & $\begin{array}{l}30 \\
99\end{array}$ & 56 & $\begin{array}{r}35 \\
108\end{array}$ \\
\hline Dopamin & 247 & $\begin{array}{l}184 \\
374\end{array}$ & 226 & $\begin{array}{l}150 \\
357\end{array}$ & 234 & $\begin{array}{l}155 \\
374\end{array}$ \\
\hline
\end{tabular}


Tab. 8

Produkt-Moment-Korrelationskoeffizienten (linke obere Hälfte Frauen - rechte untere Hälfte Männer) der bestimmten Verbindungen. Die in Klammern angegebenen Werte sind die entsprechenden Rang-Korrelationskoeffizienten

\begin{tabular}{|c|c|c|c|c|c|}
\hline Bestandteil & Adrenalin & Noradrenalin & Dopamin & Gesamtkatecholamine & Vanillinmandelsäure \\
\hline Adrenalin & 1 & $\begin{array}{c}0,016 \\
(0,017)\end{array}$ & $\begin{array}{c}0,068 \\
(0,150)\end{array}$ & $\begin{array}{c}0,366 \\
(0,263)\end{array}$ & $\begin{array}{c}0,330 \\
(0,261)\end{array}$ \\
\hline Noradrenalin & $\begin{array}{c}0,039 \\
(0,151)\end{array}$ & 1 & $\begin{array}{c}0,216 \\
(0,152)\end{array}$ & $\begin{array}{c}0,849 \\
(0,851)\end{array}$ & $\begin{array}{c}0,294 \\
(0,299)\end{array}$ \\
\hline Dopamin & $\begin{array}{c}0,298 \\
(0,321)\end{array}$ & $\begin{array}{c}0,554 \\
(0,596)\end{array}$ & 1 & $\begin{array}{c}0,269 \\
(0,273)\end{array}$ & $\begin{array}{c}0,186 \\
(0,169)\end{array}$ \\
\hline Gesamtkatecholamine & $\begin{array}{c}0,499 \\
(0,440)\end{array}$ & $\begin{array}{c}0,809 \\
(0,819)\end{array}$ & $\begin{array}{c}0,680 \\
(0,673)\end{array}$ & 1 & $\begin{array}{c}0,239 \\
(0,357)\end{array}$ \\
\hline Vanillinmandelsäure & $\begin{array}{l}0,127 \\
(0,053)\end{array}$ & $\begin{array}{c}0,583 \\
(0,562)\end{array}$ & $\begin{array}{l}0,528 \\
(0,498)\end{array}$ & $\begin{array}{c}0,579 \\
(0,536)\end{array}$ & 1 \\
\hline
\end{tabular}

Als weitere Frage interessierte, inwieweit die gemessenen Größen, die jeweils aus derselben Probe bestimmt wurden, miteinander korreliert sind. Es wurden die Produkt-Moment-Korrelationskoeffizienten und die Rang-Korrelationskoeffizienten berechnet (30). In Tabelle 8 sind die berechneten Korrelationskoeffizienten für Männer und Frauen getrennt zusammengefaßt.

Die Rang-Korrelationskoeffizienten stimmen mit den Produkt-Moment-Korrelationskoeffizienten weitgehend überein. Nach diesen Werten liegt keine Korrelation der Adrenalin- und Noradrenalinausscheidung vor. Diés erklärt sich aus der unterschiedlichen Herkunft der beiden Substanzen. Das im Urin ausgeschiedene Adrenalin stammt weitgehend aus dem Nebennierenmark und das Noradrenalin aus dem sympathischen Nervensystem (31). Die hohen Korrelationskoeffizienten für Noradrenalin und Gesamtkatecholamine sind methodisch bedingt, denn das Noradrenalin macht den Hauptteil bei der Gesamtkatecholaminbestimmung aus. Auffallend ist der Unterschied der Korrelationskoeffizienten für die Vanillinmandelsäureausscheidung mit der Noradrenalin-, Dopamin- und Gesamtkatecholaminausscheidung für Männer und Frauen. Eine schlüssige Erklärung kann nicht gegeben werden.

\section{Kasuistik}

Bei der Analyse unserer Einsendungen konnte nach relativ kurzer Zeit ein Fall mit einer Störung des Katecholaminstoffwechsels festgestellt werden ${ }^{13}$ ). Die Werte der 24-Stunden-Ausscheidung sind in folgender Tabelle wiedergegeben.

Tab. 9

24-Stunden-Ausscheidung der Katecholamine und der Vanillinmandelsäure bei einem 30 Jahre alten Patienten mit operativ gesichertem Phäochromocytom der rechten Nebenniere u. d. N. = unter der Nachweisgrenze

\begin{tabular}{|c|c|c|c|c|}
\hline $\begin{array}{c}\begin{array}{c}\text { Zeitpunkt } \\
\text { der } \\
\text { Bestimmung }\end{array} \\
\end{array}$ & $\begin{array}{l}\text { Adrenalin } \\
\mu \mathrm{g} / 24 \mathrm{Stdn}\end{array}$ & $\begin{array}{c}\text { Nor- } \\
\text { adrenalin } \\
\mu \mathrm{g} / 24 \mathrm{Stdn}\end{array}$ & $\begin{array}{c}\text { Vanillin- } \\
\text { mandelsäure } \\
\text { mg/24 Stdn }\end{array}$ & $\underset{\mu g / 24 \text { Stdn }}{\text { Dopamin }}$ \\
\hline $\begin{array}{l}8 \text { Wochen } \\
\text { präoperativ } \\
8 \text { Monate }\end{array}$ & u. d. N. & 910 & $11 ; 0$ & 276 \\
\hline postoperativ & 11,3 & 85 & 4,4 & 227 \\
\hline
\end{tabular}

13) Herrn Dr. P. Hefes, Dornbirn/Österreich möchte ich für die Zusendung der Probe danken.
Bei dem 30jährigen Patienten mit einem seit längerer Zeit bestehenden Dauerhochdruck waren sowohl die Vanillinmandelsäure- wie auch die Noradrenalinausscheidung erhöht. Operativ konnte ein Phäochromocytom der rechten Nebenniere gesichert werden.

\section{Die diagnostische Bedeutung der Katecholamin- bestimmung}

Häufig wird die Frage gestellt, ob in der Routinediagnostik zur Erkennung von Störungen des Katecholaminstoffwechsels die Bestimmung der Vanillinmandelsäure oder der Katecholamine durchgeführt werden soll. $\mathrm{K}$. Engelaran, der in einer Übersicht von 62 Fällen mit Phäochromocytom referiert, kommt zu dem Schluß, $\mathrm{da} ß$ in den meisten Fällen eine einmalige Bestimmung der Vanillinmandelsäure oder der Metanephrine ausreicht, um die Diagnose zu sichern (32). Außerdem ist die Bestimmung der Vanillinmandelsäure weniger störanfällig als die der Katecholamine. Als zusätzliche Sicherung zum Ausschluß falsch negativer Ergebnisse ist für Routineuntersuchungen die Bestimmung der Gesamtkatecholamine zu empfehlen. Die Bestimmung der Gesamtkatecholamine hat allerdings den Nachteil, $\mathrm{da} B$ sie in erster Linie das Noradrenalin erfaßt und gegenüber weniger stark erhöhten Ausscheidungen von Adrenalin und Dopamin unempfindlich ist. $\mathrm{Da}$ es sich beim Phäochromocytom meist um Noradrenalin produzierende Tumoren handelt, ist dieser Nachteil nicht so schwerwiegend. Es sei allerdings darauf hingewiesen, $\mathrm{da} B$ bei Tumoren, die sich von Neuroblasten oder Sympathocyten ableiten, die 3-O-methylierten Derivate von Dopa, Dopamin und Noradrenalin vermehrt ausgeschieden werden (33). In diesem Fall sollte man außerdem die Ausscheidung der Homovanillinsäure ermitteln.

Herrn Privatdozent Dr. Dr. Stamm, Leiter der Klinisch-chemischen Abteilung, gilt mein persönlicher Dank für die Förderung und die wertvollen Diskussionen bei der Durchführung dieser Arbeit.

Herrn Dr. E. HANSERT, Leiter der Biometrischen Abteilung des Max-Planck-Institutes für Psychiatrie, danke ich für die Beratung bei der statistischen Auswertung und Durchführung der Berechnung der Korrelationskoeffizienten.

Fräulein Jutra-MAria QUAN'E sei für ihre sorgfältige Mitarbeit gedankt. 


\section{Literatur}

1. Weil-Malherbe, H., und L. B. Bigelow, Analytic. Biochem. 22, 321 (1968). - 2. Weil-Malherbe, H., Meth. Bịochem. Analysis 16, 302 (1968). - 3. Crout, J. R., Stand. Meth. Clin. Chem. 3, 62 (1961). - 4. HÄGGENDAHL, J., Scand. J. clin. Laborat. İnvest. 14, 537 (1962). - 5. Bertler, A., A. Carlsson und E. Rosengren, Acta physiol. Scand. 44, 273 (1958). - 6. EuLER, U. S. v., und F. LishajKo, Acta physiol. Scand. 45, 122 (1959). - 7. UdENFRIEND, $\mathrm{S}$. in: Fluorescence Assay in Biology and Medicine Academic Press, New York (1964). - 8. Merrills, R. J., Nature London 193, 988 (1962). - 9. Merrills, R. J., Analytic. Biochem. 6, 272 (1963). - 10. HäGGENDAHL, J., Acta physiol. Scand. 59, 242 (1963). -11. Vochten, R. F., und, A.F. DE Schaepdryver, Experientia Basel 22, 772 (1966). - 12. Euler, U. S. v., und I. Floding, Acta physiol. Scand. 33, Suppl. 118, 45 (1955). - 13. RoBinson, R. L., und D. T. WatTs, Clin. Chem. New York 11, 986 (1965). 14. Hathaway, P. W., L. Jakor, W. G. Troyer jr. und M. D. Bogdonoff, Analytic. Biochem. 20, 466 (1967). - 15. SAMPSON, P. A., Clin. Chem. New York 13, 806 (1967). - 16. Viktoria, J. K., A. Baukal und F. W. WolfF, Analytic. Biochem. 23, 513 (1968). - 17. Mabry, C. Ch., und P. W. Warth, Amer. J. clin.
Path. 52,57 (1969). - 18. VAlori, C., C. A. Brunori, V. RenzinI und L. Corea, Analytic. Biochem. 33, 158 (1970). - 19. WrSSER, H., und D. StAMM, diese Z. 7, 631 (1969). - 20. WISSER, H., und D. Stamm, Z. analyt. Chem. (im Druck). - 21. Anton, A. H., und D. F. SAYre, J. Pharmacol. Exper. Therap. Baltimore 138, 360 (1962): - 22. KAISER, H:, Z. analyt. Chem. 209, 1 (1965). - 23. Kärkr, N. T., Acta physiol. Scand. 39, Suppl. 132 (1956). - 24. WeIl-Malmerbe, H., diese Z: 6, 161 (1964). - 25. Studnitz v., W., Klin. Wschr. 40, 163 (1962). - 26. Chang, C. C., Int. J. Neuropharmacol. 3, 643 (1964). - 27. Aschoff, J., M. FatransKa, H. GIedke, P. Doerr, D. StAMm, H. WisSer (in Vorbereitung). 28. Green, M., und G. WALKer, Clin. Chim. Acta Amsterdam 29, 189 (1970). - 29. WISSER, H., und D. STAMM, diese Z. 8, 21 (1970). - 30. WEBER, E., in: Grundriß der biologischen Statistik VEB Gustav Fischer Verlag Jena, 6. Auflage (1967). - 31. EuLER, U. S. v., S. Hellner-BjörkMaN und I. ORWEN, Acta physiol. Scand. 33, Suppl. 118, 10 (1955). - 32. Sjoerdsma, A., K. Engelman, Th. Waldmann und L. Cooperman, Ann. Int. Med. 65, 1302 (1966). 33. Gjessing, L. R., Adv. Clin. Chemistry 11, 81 (1968).

Dr. Dr. H. Wisser 8000 München 23 Kraepelinstr. 10 